\title{
ASYMPTOTIC ANALYSIS OF GROWING PLANE STRAIN TENSILE CRACKS IN ELASTIC-IDEALLY PLASTIC SOLIDS
}

\author{
W. J. Drugan, * J. R. Rice† and T-L. Sham $\ddagger$ \\ Department of Engineering Mechanics, \\ University of Wisconsin, Madison, WI 53706, U.S.A.
}

(Received 30 March 1982)

\begin{abstract}
Aastract
AN EXACT asymptotic analysis is presented of the stress and deformation fields near the tip of a quasistatically advancing plane strain tensile crack in an elastic-ideally plastic solid. In contrast to previous approximate analyses, no assumptions which reduce the yield condition, a priori, to the form of constant inplane principal shear stress near the crack tip are made, and the analysis is valid for general Poisson ratio $v$. Specific results are given for $y=0.3$ and 0.5 , the latter duplicating solutions in previous work by L. I. Slepyan, Y.C. Gao and the present authors. The crack tip field is shown to divide into five angular sectors of four different types; in the order in which these sweep across a point in the vicinity of the advancing crack, they are : two plastic sectors which can be described asymptotically (i.e., as $r \rightarrow 0$, where $r$ is distance from the crack tip) in slip-line terminology as 'constant stress' and 'centered fan' sectors, respectively; a plastic sector of nonconstant stress which cannot be described asymptotically in terms of slip lines; an elastic unloading sector; and a trailing plastic sector of the same type as that directly preceding the elastic sector. Further, these four different sector types constitute the full set of asymptotically possible solutions at the crack tip. As is known from prior work, the plastic strain accumulated by a material point passing through such a moving 'centered fan' sector is $O(\ln r)$ as $r \rightarrow 0$; it is proved in the present work that the plastic strain accumulated by a material point passing through the 'constant stress' sector ahead of a growing crack must be less singular than In $r$ as $r \rightarrow 0$. As suggested also in earlier studies, the rate of increase of opening gap $\delta$ at a point currently at a distance $r$ behind, but very near, the crack tip is given for crack advance under contained yielding by

$$
\delta=\alpha j / \sigma_{0}+\beta\left(\sigma_{0} / E\right) \dot{a} \ln (R / r)
$$

where $a$ is crack length, $\sigma_{0}$ is tensile yield strength, $E$ is Young's modulus, $J$ is the value of the $J$-integral taken in surrounding elastic material, and the parameters $\alpha$ and $R$ are undetermined by the asymptotic analysis. The exact solution for $v=0.3$ gives $\beta=5.462$, which agrees very closely with estimates obtained from finite element solutions. An approximate analysis based on use of slip line representations in all plastic sectors is outlined in the Appendix.
\end{abstract}

\section{INTRODUCTION}

INITIAL investigations of plane strain crack growth in elastic-ideally plastic materials (RICE, 1968, 1974; Cherepanov, 1974) elucidated some prominent features of the problem, most significantly that the strain possesses a weak singularity proportional to the logarithm of distance as the crack tip is approached. A full asymptotic solution for a

* (C) U.S.A. Government.

† Division of Applied Sciences, Harvard University, Cambridge, MA 02138, U.S.A.

\$Department of Mechanical Engineering, Aeronautical Engineering and Mechanics, Rensselaer Polytechnic Institute, Troy, NY 12181, U.S.A. 
growing crack in an elastic -ideally plastic solid under plane strain conditions was first obtained by SLEPYAN (1974) for a Tresca material, and solutions for a Huber-Mises material were developed later but independently by GAO (1980a) (for Poisson ratio $v=0.5$ only), and by RICE, Drugan and SHAM (1980). The latter work extended and corrected, by inclusion of an elastic unloading zone, the earlier RICE and SORENSEN (1978) analysis, which assumed the stationary Prandtl configuration to be maintained at a growing crack. However, as commented by RICE (1982), some errors remain in the Rice, Drugan and Sham results presented for $v \neq 0.5$; the fully correct solution is given here. Slepyan and Gao treated the steady-state crack growth problem only; Rice, Drugan and Sham considered general, non-steady growth and amplified their asymptotic results by estimating some parameters that are left undetermined in the asymptotic analysis, via comparison with a numerical finite element solution.

The present paper provides an exact asymptotic analysis, within 'small strain' theory (neglect of deformation effects on stress measures and equilibrium equations), of the stress and deformation fields near the tip of a growing tensile crack under plane strain conditions in an elastic-ideally plastic isotropic Huber-Mises solid for general $v$, obtaining specific results for the $v=0.3$ and $v=0.5$ cases. The analysis presented utilizes a framework developed by RICE (1982) for determining the class of stress and deformation rate fields which are admissible, asymptotically, at a quasi-statically advancing crack tip. That work was done for arbitrary deformation modes (plane stress, plane and anti-plane strain; tension and/or shear) in materials of arbitrary yield condition and associated flow rule, including anisotropic response. Here we present concisely the results of Rice's asymptotic analysis for the present case of plane strain, isotropic response obeying the Huber-Mises yield condition and the (associated) Prandtl-Reuss flow rule. We then show how the various near-tip sectors are assembled to solve the tensile crack problem.

Figure 1 illustrates the geometry of the problem. Cartesian coordinates $x_{1}, x_{2}, x_{3}$ are chosen so that $x_{1}$ points in the direction of crack growth, with $a$ being the measure of crack length, and $x_{3}$ is parallel to the crack front. Polar coordinates $r, \theta$ lie in the $x_{1}, x_{2}$ plane, are centered at the crack tip and move with it through the material, $\theta$ being measured from the line ahead of the crack. This polar system has the corresponding unit vectors $\mathbf{e}$ in the radial direction and $\mathbf{h}$ in the angular direction. Evidently then

$$
\partial r / \partial x_{\alpha}=e_{\alpha}, \quad \partial \theta / \partial x_{\alpha}=h_{\alpha} / r
$$

where

$$
e_{1}=h_{2}=\cos \theta, \quad e_{2}=-h_{1}=\sin \theta, \quad e_{3}=h_{3}=0
$$

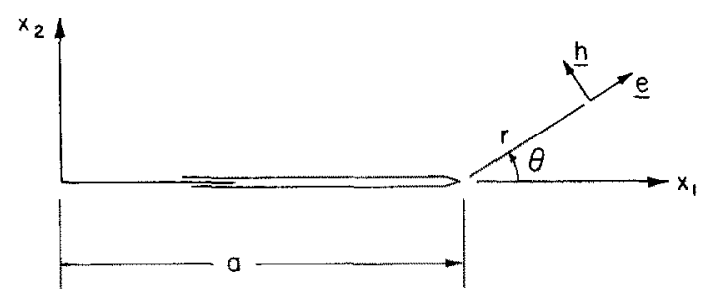

FIG. 1. Cartesian coordinates $x_{1}, x_{2}, x_{3}$ are fixed in the body; polar coordinates $r, \theta$ are centered at the tip and move with it through the material during crack growth. 
Greek indices $\alpha, \beta, \gamma, \delta$, used here and subsequently, have range 1,2 only; Latin indices $i, j, k, l$ will be understood to have the range $1,2,3$; both types of index follow the summation convention and are always understood to index Cartesian (but never polar) components of various vectors or tensors.

\section{Formulation for Plane Strain Asymptotic Analysis}

In this section the equations describing plane strain deformation of an isotropic elastic-ideally plastic solid are presented in the forms to which they reduce very near the tip of a quasi-statically extending crack, by specializing the results of RICE (1982). For plane strain deformation, $u_{3}=0, \varepsilon_{3 i}=0$ and $\sigma_{3 \alpha}=0$, where $u_{i}$ are the displacement components, $\varepsilon_{\mathrm{ij}}$ is the infinitesimal strain tensor and $\sigma_{i j}\left(=\sigma_{j i}\right)$ is the stress tensor.

\subsection{Equilibrium}

The equations of plane equilibrium,

$$
\partial \sigma_{\alpha \beta} / \partial x_{\beta}+f_{\alpha}=0
$$

phrased in terms of the crack tip polar coordinate system via (1.1), are

$$
\left(\partial \sigma_{\alpha \beta} / \partial \theta\right)\left(h_{\beta} / r\right)+\left(\partial \sigma_{\alpha \beta} / \partial r\right) e_{\beta}+f_{\alpha}=0
$$

where $f_{\alpha}$ is the body force vector. Multiplying (2.1) by $r$, it is evident that $r\left(\partial \sigma_{\alpha \beta} / \partial r\right) e_{\beta} \rightarrow 0$ and $r f_{\alpha} \rightarrow 0$ as $r \rightarrow 0$, the former since stresses are necessarily bounded in the elasticideally plastic model being treated. Thus equilibrium very near the crack tip $(r \rightarrow 0)$ requires

where

$$
h_{\alpha} \sigma_{\alpha \beta}^{\prime}=0
$$

$$
\sigma_{\alpha \beta}^{\prime} \equiv \lim _{r \rightarrow 0}\left\{\partial \sigma_{\alpha \beta}(r, \theta, t) / \partial \theta\right\}
$$

and thus restricts only the $\theta$-variations of stress components. Using (1.2) these equilibrium conditions can be written out as

$$
e_{2} \sigma_{11}^{\prime}=e_{1} \sigma_{21}^{\prime}, \quad e_{2} \sigma_{12}^{\prime}=e_{1} \sigma_{22}^{\prime},
$$

or in terms of the polar coordinate stress components as

$$
\sigma_{r r}-\sigma_{\theta \theta}+\sigma_{r \theta}^{\prime}=0, \quad 2 \sigma_{r \theta}+\sigma_{\theta \theta}^{\prime}=0 .
$$

Expression of the three independent components $(11,12,22)$ of $\sigma_{\alpha \beta}^{\prime}$ in terms of any one is possible by (2.3), and this enables one to show (RICE, 1982) that the scalar product of an arbitrary tensor $H_{i j}$ with $\sigma_{i j}^{\prime}$ is expressible as

$$
\sigma_{i j}^{\prime} H_{i j}=\left(\sigma_{12}^{\prime} / e_{1} e_{2}\right) H_{r r}+\sigma_{33}^{\prime} H_{33}=\left(\sigma_{11}^{\prime}+\sigma_{22}^{\prime}\right) H_{r r}+\sigma_{33}^{\prime} H_{33},
$$

whenever $\sigma_{i j}$ satisfies the equilibrium equations, where $H_{r r} \equiv e_{i} e_{j} H_{i j}$ is the radial, ondiagonal component of $H_{i j}$. (Note that the repeated $r$ indices do not imply summation : $r$ denotes radial direction.) 


\subsection{Elastic-plastic stress-strain relations}

Material points near the tip of a growing crack are subjected to a highly nonproportional loading history, necessitating the use of an incremental plasticity theory which is expressed here in terms of rates. The rate of deformation is defined as

$$
D_{i j}=\frac{1}{2}\left(\partial v_{i} / \partial x_{j}+\partial v_{j} / \partial x_{i}\right)
$$

where $v_{i}$ are the components of material velocity. For material which is currently experiencing elastic-plastic deformation, the rate of deformation is assumed to be given by the Prandtl-Reuss flow rule

$$
D_{i j}=D_{i j}^{\mathrm{e}}+D_{i j}^{\mathrm{p}}=\frac{1+v}{E} \dot{\sigma}_{i j}-\frac{v}{E} \delta_{i j} \dot{\sigma}_{k k}+\Lambda s_{i j}
$$

where $E$ is Young's modulus, $v$ is Poisson's ratio, the superposed dot denotes time rate at a material point, $\Lambda \geqslant 0$ is an undetermined parameter and $s_{i j}=\sigma_{i j}-\delta_{i j} \sigma_{k k} / 3$ is the deviatoric stress tensor. For material which currently experiences only elastic deformation, whether or not it has previously deformed plastically, (2.7) applies with $\Lambda=0$. Writing $\sigma_{i j}=\sigma_{i j}(r, \theta, t)$, the stress rate may be calculated as

$$
\begin{aligned}
\dot{\sigma}_{i j} & =\left(\partial \sigma_{i j} / \partial \theta\right) \dot{\theta}+\left(\partial \sigma_{i j} / \partial r\right) \dot{r}+\partial \sigma_{i j} / \partial t \\
& =\left(\partial \sigma_{i j} / \partial \theta\right)\left(e_{2} \dot{a} / r\right)-\left(\partial \sigma_{i j} / \partial r\right)\left(e_{1} \dot{a}\right)+\partial \sigma_{i j} / \partial t,
\end{aligned}
$$

since due to crack growth and hence translation of the crack tip polar coordinate system, $\dot{\theta}=e_{2} \dot{a} / r$ and $\dot{r}=-e_{1} \dot{a}$. Multiplying (2.8) by $r$ and assuming further that $r \partial \sigma_{i j}(r, \theta, t) / \partial t \rightarrow 0$ as $r \rightarrow 0$, one obtains

$$
r \dot{\sigma}_{i j} \rightarrow \sigma_{i j}^{\prime} e_{2} \dot{a} \text { as } r \rightarrow 0
$$

This result shows also that in near-tip angular sectors in which Cartesian stress components vary with $\theta$, stress rates and thus elastic strain rates are singular as $\dot{a} / r$. Use of (2.9) permits the Prandt1-Reuss flow rule (2.7) to be written in the asymptotic form

$$
r\left(D_{i j}-\Lambda s_{i j}\right) \rightarrow\left[\frac{1+v}{E} \sigma_{i j}^{\prime}-\frac{v}{E} \delta_{i, j} \sigma_{k k}^{\prime}\right] \dot{a} \sin \theta \quad \text { as } \quad r \rightarrow 0
$$

\subsection{Yield condition}

The Huber-Mises yield condition is assumed, namely

$$
f\left(\sigma_{i j}\right)=s_{i j} s_{i j} / 2-k^{2}=0
$$

where $k$ is the shear strength. The analysis is clarified by adopting a differential form of this yield condition

$$
s_{i j}\left(\partial \sigma_{i j} / \partial \theta\right)=0
$$

which also holds in all plastic regions of the body. As $r \rightarrow 0$, this condition requires

$$
s_{i j} \sigma_{i j}^{\prime}=0
$$


which can be rewritten via (2.5) as

$$
\left(\sigma_{11}^{\prime}+\sigma_{22}^{\prime}\right) s_{r r}+\sigma_{33}^{\prime} s_{33}=0
$$

to be satisfied in all near-tip angular regions of plastic response.

\subsection{Restrictions on the asymptotic deformation rate fields}

2.4.1 Plane strain condition. The plane strain condition $\varepsilon_{33}=0$ (or $D_{33}=0$ ) permits determination of out-of-plane deformation quantities. Since $\varepsilon_{33}=\varepsilon_{33}^{e}+\varepsilon_{33}^{p}$, the condition requires that

$$
\varepsilon_{33}^{\mathrm{p}}=-\varepsilon_{33}^{\mathrm{e}}=-\left[\sigma_{33}-v\left(\sigma_{11}+\sigma_{22}\right)\right] / E,
$$

showing that $\varepsilon_{33}^{\mathrm{p}}$ must be bounded everywhere. Furthermore, $D_{33}^{\mathrm{p}}$ can be determined by taking the material time derivative of $(2.15)$, which, via (2.9), becomes

$$
r D_{33}^{\mathrm{p}}=r \dot{\varepsilon}_{33}^{\mathrm{p}} \rightarrow-\left[\sigma_{33}^{\prime}-v\left(\sigma_{11}^{\prime}+\sigma_{22}^{\prime}\right)\right] \dot{a} \sin \theta / E \quad \text { as } \quad r \rightarrow 0 .
$$

Thus $D_{33}^{\mathrm{p}} \sim \dot{a} / r$ as $r \rightarrow 0$ even though we have shown $\varepsilon_{33}^{\mathrm{p}}$ to be bounded. This suggests that if $D_{i j}^{\mathrm{p}} \sim \dot{a} / r$ as $r \rightarrow 0$ in a plastically deforming sector, the contribution to the corresponding $\varepsilon_{i j}^{\mathrm{p}}$ from that sector will be bounded (except if $D_{i j}^{\mathrm{p}} \sim \dot{a} / r$ as $r \rightarrow 0$ along $\theta=0$ ). That this is indeed true is easily proved by integrating $D_{i j}^{\mathrm{p}}=\dot{a} F_{i j}(\theta) / r\left[F_{i j}(\theta)\right.$ arbitrary] at a material point when $a$ is increasing continuously with applied loading; this integration is accomplished (RICE, 1974) by using $\dot{\theta}=e_{2} \dot{a} / r$ to substitute for $\dot{a}$ (when $\theta \neq 0$ ):

$$
\varepsilon_{i j}^{\mathrm{p}}=\int^{t} D_{i j}^{\mathrm{p}} \mathrm{d} \tau=\int^{t}\left[\dot{a} F_{i j}(\theta) / r\right] \mathrm{d} \tau=\int^{\theta}\left[F_{i j}(\phi) / \sin \phi\right] \mathrm{d} \phi
$$

which is evidently bounded as $r \rightarrow 0$.

2.4.2 Consequences of plastic incompressibility and the yield condition. Deformation rates near the growing crack tip are given by the asymptotic form (2.10) of the Prandt 1 Reuss flow rule, which in plastically deforming sectors contains the undetermined parameter $\Lambda$. Restrictions on the near-tip deformation rate field which do not involve this unknown parameter are sought. RICE (1982) showed that two such restrictions can be found, which, in the present case, specialize to

$$
\begin{gathered}
\partial v_{\theta} / \partial \theta+\partial\left(r v_{r}\right) / \partial r \rightarrow(1-2 v) \sigma_{k k}^{\prime} \dot{a} \sin \theta / E \text { as } r \rightarrow 0, \\
r\left(\partial v_{r} / \partial r\right)\left(\sigma_{11}^{\prime}+\sigma_{22}^{\prime}\right) \rightarrow\left[\left(\sigma_{11}^{\prime}+\sigma_{22}^{\prime}\right)^{2}-2 v\left(\sigma_{11}^{\prime}+\sigma_{22}^{\prime}\right) \sigma_{33}^{\prime}\right. \\
\left.+\left(\sigma_{33}^{\prime}\right)^{2}\right] \dot{a} \sin \theta / E \text { as } r \rightarrow 0 .
\end{gathered}
$$

Condition (2.17) arises from plastic incompressibility (inherent in the adoption of the Prandtl-Reuss flow rule), and is obtained by taking the trace of (2.10). Condition (2.18) results from first multiplying (2.7) by $r \partial \sigma_{i j} / \partial \theta$, noting that the term $r \Lambda s_{i j} \partial \sigma_{i j} / \partial \theta=0$ always (because $\Lambda=0$ in elastic regions and because (2.12) holds in plastic regions), and then by letting $r \rightarrow 0$ and making repeated use of (2.5). 


\section{Characterization of Near-tip Angular Sectors}

\subsection{Stress and deformation-rate fields in currently elastic sectors}

The governing equations for an asymptotic sector whose current response is purely elastic are the equilibrium equations (2.3), the plane strain condition $D_{33}=D_{33}^{\mathrm{e}}=0$, the deformation rate expression (2.10) with $\Lambda=0$, and since the formulation is in terms of deformation rate, the requirement that the deformation rate field be compatible with a velocity field,

$$
\partial^{2} D_{22} / \partial x_{1}^{2}+\partial^{2} D_{11} / \partial x_{2}^{2}=2 \partial^{2} D_{12} / \partial x_{1} \partial x_{2}
$$

Since $\sigma_{i j}$ varies with $\theta$ at the tip and $D_{i j}$ is proportional to $\dot{\sigma}_{i j}$ in an elastic sector, it is evident (see 2.10) that $r D_{i j}$ approaches a bounded function of $\theta$ at the tip, i.e., that $D_{i j} \sim \dot{a} F_{i j}(\theta) / r$ as $r \rightarrow 0$. RiCE (1982) showed [equivalent results were first obtained by SLEPYAN (1974), and later, but independently, by GAO (1980a) and by RICE, DRUGAN and SHAm (1980)] that the most general expression of this type satisfying (3.1) must be such that $F_{r r}\left(=e_{i} e_{j} F_{i j}\right)$ satisfy $\mathrm{d}^{2} F_{r r} / \mathrm{d} \theta^{2}+F_{r r}=0$, and that the most general possible form of velocity and stress fields in a currently elastic sector is therefore

$$
\begin{aligned}
v_{1} & =\dot{a} \mathrm{~A}_{1} \ln |r \sin \theta / \bar{R}|-\dot{a} \int\left[F_{11}(\theta) / \sin \theta\right] \mathrm{d} \theta, \\
v_{2} & =\dot{a} \mathrm{~A}_{2} \ln |r \cos \theta / \bar{R}|+\dot{a} \int\left[F_{22}(\theta) / \cos \theta\right] \mathrm{d} \theta,
\end{aligned}
$$

to within arbitrary rigid motion, where $\mathrm{A}_{1}$ and $\mathrm{A}_{2}$ are constants, $\bar{R}$ is an undetermined length parameter,

$$
\begin{aligned}
& F_{11}(\theta)=\left(\cos ^{2} \theta-v\right)\left(\mathrm{A}_{1} \cos \theta+\mathrm{A}_{2} \sin \theta\right) /(1-v), \\
& F_{22}(\theta)=\left(\sin ^{2} \theta-v\right)\left(\mathrm{A}_{1} \cos \theta+\mathrm{A}_{2} \sin \theta\right) /(1-v),
\end{aligned}
$$

and

$$
\begin{aligned}
M \sigma_{11} & =4 \mathrm{~A}_{1} \ln |\sin \theta|+\mathrm{A}_{1} \cos 2 \theta+\mathrm{A}_{2}(2 \theta+\sin 2 \theta)+\mathrm{C}_{11}, \\
M \sigma_{12} & =\mathrm{A}_{1}(2 \theta+\sin 2 \theta)-\mathrm{A}_{2} \cos 2 \theta+\mathrm{C}_{12}, \\
M \sigma_{22} & =-\mathrm{A}_{1} \cos 2 \theta+\mathrm{A}_{2}(2 \theta-\sin 2 \theta)+\mathrm{C}_{22}, \\
\sigma_{33} & =v\left(\sigma_{11}+\sigma_{22}\right)+\mathrm{d},
\end{aligned}
$$

where $M \equiv 4\left(1-v^{2}\right) / E$ and $\mathrm{C}_{11}, \mathrm{C}_{12}, \mathrm{C}_{22}$ and $\mathrm{d}$ are constants of integration.

\subsection{Classification and analysis of plastically deforming sectors}

As noted by RICE (1982), it is convenient to divide consideration of plastically deforming near-tip sectors into two classes: sectors in which $s_{33} \rightarrow 0$ as $r \rightarrow 0$, and sectors in which $s_{33} \neq 0$ at $r=0$. (This analysis was developed to permit exact asymptotic treatment of the general case of elastic compressibility; for the special case of elastic, as well as plastic, incompressibility $(v=1 / 2), s_{33}=0$ everywhere.)

3.2.1 Plastically deforming sectors in which $\mathrm{s}_{33} \rightarrow 0$ as $\mathrm{r} \rightarrow 0$. RICE and TRACEY (1973) showed that in asymptotic plastic sectors in which $s_{33} \rightarrow 0$ as $r \rightarrow 0$, the equilibrium 
equations (2.4) and the Huber-Mises yield condition (2.11) permit only two types of plastic sector. This is obvious immediately from $(2.14)$, which now reduces to $\left(\sigma_{11}^{\prime}+\sigma_{22}^{\prime}\right) s_{r r}=0$ so that solutions are of the types (i) $\sigma_{11}^{\prime}+\sigma_{22}^{\prime}=0$ and (ii) $s_{r r}=0$. After a little analysis these are seen easily to correspond to:

(i) constant stress sectors, in which $\sigma_{11}=$ constant, $\sigma_{12}=\sigma_{21}=$ constant, and $\sigma_{22}=$ constant, $\sigma_{33}=\left(\sigma_{11}+\sigma_{22}\right) / 2$, where these constants must satisfy the yield condition, and

(ii) centered fan sectors, in which

$$
\begin{aligned}
& \sigma_{r \theta}= \pm \mathrm{k}, \\
& \sigma_{r r}=\sigma_{\theta \theta}=\sigma_{33}=\mathrm{constant} \mp 2 \mathrm{k} \theta .
\end{aligned}
$$

The sectors are so named because their (asymptotic) stress fields are the same as those of constant stress and centered fan sectors, respectively, in plane strain rigid-plastic slip line theory.

Following RICE (1982), the structure of the velocity field in a centered fan sector can be determined from (2.17) and (2.18) since $\sigma_{i j}^{\prime}$ are completely specified by (3.5) [choosing the sign appropriate for a crack under Mode I tension, i.e., $\sigma_{33}^{\prime}=\left(\sigma_{11}^{\prime}+\sigma_{22}^{\prime}\right) / 2=$ $-2 k]$. Regarding (2.17) and (2.18) as equalities sufficiently close to the crack tip, they may be integrated to give (apart from rigid motions)

$$
\begin{aligned}
v_{r}= & (5-4 v)(\mathrm{k} / E) \dot{a} \sin \theta \ln (\bar{R} / r)+\partial f(\theta, t) / \partial \theta, \\
v_{\theta}= & -(5-4 v)(\mathrm{k} / E) \dot{a}[(1 / \sqrt{ } 2)-\cos \theta] \ln (\bar{R} / r) \\
& +[(5-4 v)-6(1-2 v)](\mathrm{k} / E) \dot{a}[(1 / \sqrt{ } / 2)-\cos \theta]-f(\theta, t),
\end{aligned}
$$

where $f(\theta, t)$ is a function of integration that is undetermined by the asymptotic analysis; (3.6) are valid for a fan beginning at $\theta=\pi / 4$, which will be the case in our solutions. These equations permit determination of the deformation rates to $O(1 / r)$ in a centered fan sector which begins at $\theta=\pi / 4$ :

$$
\begin{aligned}
D_{r r}= & \partial v_{r} / \partial r=-(5-4 v)(\mathrm{k} / E)(\dot{a} / r) \sin \theta, \\
D_{\theta \theta}= & (1 / r)\left(\partial v_{\theta} / \partial \theta+v_{r}\right)=[(5-4 v)-6(1-2 v)](\mathrm{k} / E)(\dot{a} / r) \sin \theta, \\
D_{r \theta}= & (1 / 2 r)\left(\partial v_{r} / \partial \theta-v_{\theta}\right)+(1 / 2)\left(\partial v_{\theta} / \partial r\right) \\
= & {[(5-4 v) / \sqrt{ } 2](\mathrm{k} / E)(\dot{a} / 2 r) \ln (\bar{R} / r) } \\
& +6(1-2 v)(\mathrm{k} / E)(\dot{a} / 2 r)[(1 / \sqrt{ } 2)-\cos \theta]+(1 / 2 r)\left[\partial^{2} f(\theta, t) / \partial \theta^{2}+f(\theta, t)\right], \\
D_{3 i}= & 0 .
\end{aligned}
$$

Rice and Sorensen (1978) and RiCe, Drugan and Sham (1980) have calculated the velocities and deformation rates in such a centered fan sector, and although their forms are the same as those presented here, their coefficients are incorrect due to an erroneous limit calculation, as discussed by RICE (1982). Namely, they have $2(2-v)$ instead of the correct value of $(5-4 v)$, and in their expressions a factor of 4 precedes $(1-2 v)$, while the correct factor is 6 . [Note that for $v=1 / 2,(5-4 v)=2(2-v)$ and $(1-2 v)=0$, meaning that the aforementioned results reduce to the correct expressions in the case $v=1 / 2$.] 
3.2.2 Plastically deforming sectors in which $\mathrm{s}_{33} \neq 0$ at $\mathrm{r}=0$. In such sectors the plane strain condition $D_{33}=0$ facilitates immediate determination of the parameter $\Lambda$ from the 33 component of $(2.7)$

$$
\Lambda=-\left[\dot{\sigma}_{33}-v\left(\dot{\sigma}_{11}+\dot{\sigma}_{22}\right)\right] /\left(E s_{33}\right)
$$

so that the in-plane deformation rates satisfy

$$
\begin{aligned}
& r D_{\alpha \beta} \rightarrow\left[(1+v) \sigma_{\alpha \beta}^{\prime}-v\left(\delta_{\alpha \beta}-s_{\alpha \beta} / s_{33}\right) \sigma_{\gamma \gamma}^{\prime}\right. \\
& \left.\quad-\left(v \delta_{\alpha \beta}+s_{\alpha \beta} / s_{33}\right) \sigma_{33}^{\prime}\right] \dot{a} \sin \theta / E \quad \text { as } \quad r \rightarrow 0 .
\end{aligned}
$$

It is therefore evident that $D_{\alpha \beta} \sim \dot{a} / r$ as $r \rightarrow 0$, so that by the arguments of Section 2.4.1, only bounded contributions to the plastic strain will accrue from $s_{33} \neq 0$ sectors. RicE (1982) showed, through an argument analogous to that preceding (3.2), that for (3.9) to be compatible, that is, to satisfy (3.1), the stresses must satisfy, as $r \rightarrow 0$,

$$
\left[1+v\left(s_{r r} / s_{33}\right)\right]\left(\sigma_{r r}^{\prime}+\sigma_{\theta \theta}^{\prime}\right)-\left[v+\left(s_{r r} / s_{33}\right)\right] \sigma_{33}^{\prime}=\left[\mathbf{B}_{1} \cos \theta / \sin \theta+\mathbf{B}_{2}\right] E
$$

where $B_{1}$ and $B_{2}$ are constants. Thus the four independent, non-zero stress components in a sector with $s_{33} \neq 0$ are governed by four first-order ordinary differential equations as $r \rightarrow 0$ : the equilibrium conditions (2.4), the yield condition (2.14), and the compatibility condition (3.10). If $\mathrm{B}_{1}=\mathrm{B}_{2}=0$, it is straightforward to prove that the only solution form to these equations is $\sigma_{i j}=$ constant; for $\left(B_{1}, B_{2}\right)$ different from $(0,0)$, a closed form solution to these equations could not be found, necessitating numerical solution. The near-tip velocities in an $s_{33} \neq 0$ sector were shown by RICE (1982) also to have the forms (3.2), with $\left(\mathrm{B}_{1}, \mathrm{~B}_{2}\right)$ replacing $\left(\mathrm{A}_{1}, \mathrm{~A}_{2}\right)$ and with $F_{11}(\theta)$ and $F_{22}(\theta)$ given not by (3.3) but, rather, by equating the 11 and 22 components of the right side of (3.9) to $\dot{a} F_{11}(\theta)$ and $\dot{a} F_{22}(\theta)$, respectively.

\section{Exact Asymptotic Solutions for Crack Tip Fields}

\subsection{General requirements}

An acceptable solution for the asymptotic stress and deformation fields near a crack tip must consist of a configuration of angular sectors, composed from the class of possible sectors enumerated in Section 3, and meeting certain symmetry, continuity and boundary conditions. In particular, since we consider Mode I (tension) loading, symmetry about the crack line requires

$$
\sigma_{r r}^{\prime}(0)=\sigma_{\theta \theta}^{\prime}(0)=\sigma_{33}^{\prime}(0)=v_{r}^{\prime}(0)=0, \quad \sigma_{r \theta}(0)=v_{\theta}(0)=0 .
$$

Tractions (i.e., $\sigma_{r \theta}$ and $\left.\sigma_{\theta \theta}\right)$ and displacements $\left(u_{1}, u_{2}\right)$ must be fully continuous along radial sector boundaries, and the crack faces must be traction-free,

$$
\sigma_{r o}(\pi)=\sigma_{\theta 0}(\pi)=0 \text {. }
$$

Furthermore, in all regions of ongoing plastic deformation, the resulting stress and deformation fields must produce non-negative plastic work, $\Lambda \geqslant 0$.

We first review the assembly of sectors when $v=1 / 2$. Then, following a critical observation by GAO (1981) (whose work in other respects we disagree with subsequentiy), we show the assembly for $v<1 / 2$ and give specific results for $v=0.3$. 


\subsection{Exact solutions for $v=0.5$}

Asymptotic solutions of the near-tip fields for the special case of $v=0.5$ (full incompressibility) were obtained by RICE (1967) for the stationary crack, and independently by SLEPYAN (1974) (for a Tresca material, which coincides with a HuberMises material when $v-0.5)$, GAo (1980a) and Rice, Drugan and Sham (1980) for the growing crack. The near-tip field is in general comprised of only three types of asymptotic sector in this case since $s_{33}=0$; the yield condition (2.11) reduces to

$$
\left(\sigma_{\theta \theta}-\sigma_{r r}\right)^{2} / 4+\sigma_{r \theta}^{2}=k^{2} .
$$

The two possible types of asymptotic plastic sector are centered fan and constant stress. The stress fields in these sector types are as given in Section 3.2.1 whether the crack is stationary or growing quasi-statically, since in both cases these stress fields are statically determinate, being governed by equilibrium (2.4), yield (4.3), and $s_{33}=0$. The stress field in an elastic sector near a stationary crack may in general be of different form from that in an elastic sector near a growing crack.

4.2.1 Stationary crack solution. If in addition to the requirements of Section 4.1 , it is assumed that yielding completely surrounds the stationary crack tip (i.e., no elastic sectors as $r \rightarrow 0$ ) a possible asymptotic solution is given by the Prandtl field of Fig. 2 (RICE, 1967). Regions A and C are constant stress sectors, and Region B is a centered fan sector. This is the only possible near-tip solution for a stationary crack with Mode I tension loading ahead of the crack $\left(\sigma_{22}>0\right.$ on $\left.\theta=0\right)$, if attention is limited to fully continuous stress fields (i.e., $\sigma_{r r}$ continuous as well as $\sigma_{r \theta}$ and $\sigma_{\theta \theta}$ ) and if it is assumed that yield is met at all angles about the tip. The Prandtl field seems to be reasonably substantiated as the limiting asymptotic field for well-contained yielding by its close correlation to the finite-element numerical small-scale yielding results of LEVY et al. (1971), RICE and TRACEY (1973) and many subsequent investigators, and by its status as the non-hardening limit of the HuTCHINSON (1968) and RICE and ROSENGREN (1968) singularities for power-law strain hardening materials. On the other hand, many solutions are known for the general yielding range from plastic limit analysis (e.g., MCClinToCK, 1971); these do not always show crack tip fields identical to the Prandtl field and, indeed, in cases for which they differ one observes sub-yicld sectors and possible discontinuities in $\sigma_{r r}$.

4.2.2 Growing crack solution. As discussed above, the admissible forms of the stress fields in near-tip plastic sectors are the same for stationary and growing cracks when $v=0.5$. Thus if it is assumed, as was done for the stationary crack, that material at all angles about the crack tip is deforming plastically, one might expect the same Prandtl

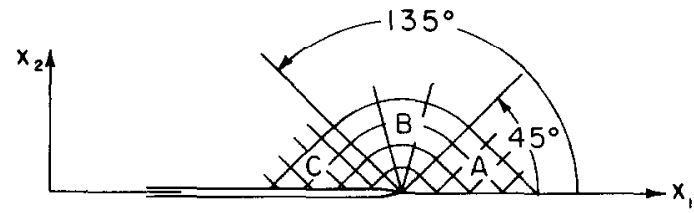

FIG. 2. The Prandtl stress field, represented in terms of slip lines, as a near-tip solution for a stationary crack under tensile loading. 
field to persist at a growing crack tip. A number of previous investigators (RICE, 1968, 1974; Cherepanov, 1974 ; RICE and SORENSEN, 1978) did make this assumption, failing to notice, as pointed out by RICE, DRUGAN and SHAM (1980), that the deformation field associated with such a moving Prandtl configuration produces a line of negative plastic work at $\theta=3 \pi / 4$, the boundary between Regions B and C in Fig. 2. The radial velocity component on the fan side of this boundary, from (3.6), is $v_{r} \sim-\ln r \rightarrow+\infty$ as $r \rightarrow 0$, while on the constant stress side $v_{r}$ is necessarily bounded. Thus this velocity jump corresponds to a negative shearing, but $\sigma_{r 0}$ is positive on the discontinuity line, resulting in negative plastic work. Consequently the Prandtl configuration is ruled out for a growing crack due to the positive plastic work requirement.

Now since the Prandtl field is the unique asymptotic solution corresponding to a fully continuous stress field under the assumption of plastic response at all angles about the crack tip, and since allowing tangential stress $\left(\sigma_{r r}\right)$ discontinuities would not eliminate the situation of a fan bordered at the rear by a constant stress sector, it is evident (as in SlePYan, 1974; GaO, 1980a; Rice, Drugan and Sham, 1980) that the assumption of continued plastic response at all angles about the crack tip must be relaxed for the growing crack. An additional observation is that the Prandtl configuration exists, apparently, under contained yielding conditions, for increments of load applied before crack growth begins and also after it stops. Thus a solution for the growing crack might be expected plausibly to resemble somewhat the Prandtl configuration while eliminating that configuration's negative plastic work region.

These considerations of non-negative plastic work imply the necessity of a sector of currently elastic response bordering the rear of a moving centered fan sector. Such an elastic sector cannot extend all the way to the crack flank since, referring to (3.4), $\sigma_{11} \sim$ $-\ln |\sin \theta|$ as $\theta \rightarrow \pi$ (attempted solution of this configuration shows $A_{1} \neq 0$ ), indicating that yielding will again occur before $\theta$ attains the value $\pi$. Another way to observe the necessity of a zone of further plasticity adjacent to $\theta=\pi$ is to note (RICE, DRUGAN and SHAM, 1980) that a moving fan produces $\varepsilon_{11}^{p} \rightarrow-\infty$ as $r \rightarrow 0$; this requires a trailing zone of reversed yielding because otherwise a material particle would emerge from the crack tip region possessing plastic strain $\varepsilon_{11}^{\mathrm{p}}$ varying with $x_{2}$ and becoming unbounded as $x_{2} \rightarrow 0$, hence producing residual stress $\sigma_{11}$ in excess of yield. Thus the form of asymptotic configuration suggested by these considerations is a modified Prandtl configuration, possessing a sector of currently elastic response interposed between the centered fan and trailing constant stress sector, Fig. 3. A solution having this form does indeed exist, with $\theta_{1}=112.08^{\circ}$ and $\theta_{2}=162.10^{\circ}$. Figure 3 has been drawn to correspond to these angles. This solution was determined independently by SLEPYAN (1974) for a Tresca material (which coincides with a Huber-Mises material when

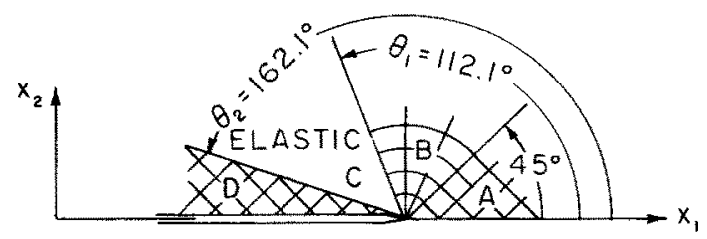

FIG. 3. This modified Prandtl stress field is an exact solution of the near-tip fields for a growing tensile crack in fully incompressible $(v=0.5)$ material. 
$v=0.5$ ), by Gao (1980a) and by Rice, Drugan and Sham (1980). It is not known whether the modified Prandtl field of Fig. 3 is the only asymptotic growing crack solution possessing full stress continuity for $v=0.5$, although we have been able to rule out several other assemblies of angular sectors.

More results for the case $v=0.5$ are given subsequently.

\subsection{Exact growing crack solution for $v<0.5$}

4.3.1 General analysis. The region near the tip of a growing plane strain crack in an isotropic elastic-ideally plastic solid with $v<0.5$ may in general be comprised of the four types of asymptotic angular sectors discussed in Section 3 : sectors of purely elastic current material response; plastic sectors of either centered fan or constant stress type, where in both cases $s_{33} \rightarrow 0$ as $r \rightarrow 0$; and non-singular plastic sectors in which $s_{33} \neq 0$ at $r=0$. An acceptable solution must consist of a configuration containing some or all of these sector types which satisfies the requirements of Section 4.1; we make the additional assumption that the tangential stresses are also continuous, so that full continuity of all stress components is required.

It is anticipated that the solution sought will have many features similar to the exact $v=0.5$ growing crack solution, since it must approach that solution as $v \rightarrow 0.5$. A configuration identical to that of the $v=0.5$ solution, Fig. 3, cannot be a solution for the general $v<0.5$ case, since continuity of $s_{33}$ across both boundaries of the elastic unloading sector cannot be met. The configuration in Fig. 4 was first hypothesized. It differs from the configuration of Fig. 3 in that the trailing plastic constant stress sector is replaced by a plastic $s_{33} \neq 0$ sector. This change permitted a solution with a fully continuous stress field satisfying the non-negative plastic work condition everywhere. However, as was pointed out by $\mathrm{GAO}_{\mathrm{AO}}(1981)$, this solution possesses the unacceptable feature that the stress field in the elastic unloading sector violates the yield condition in a small angular region directly behind the fan. This fact necessitates the introduction of a fifth angular sector, of the plastic $s_{33} \neq 0$ type (this plastic sector could not be of the constant stress type due to non-negative work requirements, see Section 4.2.2), directly following the centered fan region; this configuration is illustrated in Fig. 5. A solution possessing fully continuous stresses and satisfying all of the requirements delineated in Section 4.1 (and for which the elastic sector stress field nowhere violates yield) has been found having the configuration of Fig. 5. The means of determining this solution will now be discussed. GAO (1981) proposed a solution with this same configuration but, as

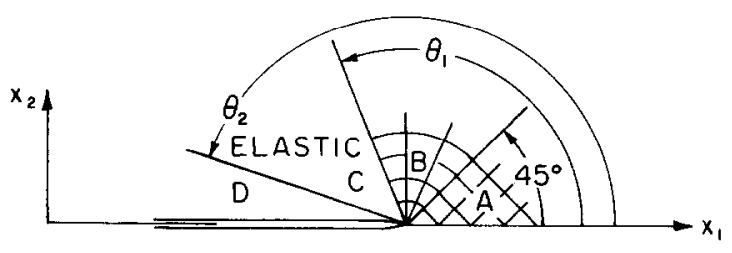

FIG. 4. This configuration was first hypothesized as a potential solution configuration for the $v<0.5$ general case, since it permits a solution possessing a fully continuous stress field which produces positive plastic work everywhere. However, this configuration must be modified since the yield condition is violated for $\theta$ in the clastic unloading sector, $\mathrm{C}$, near $\theta_{1}$. 


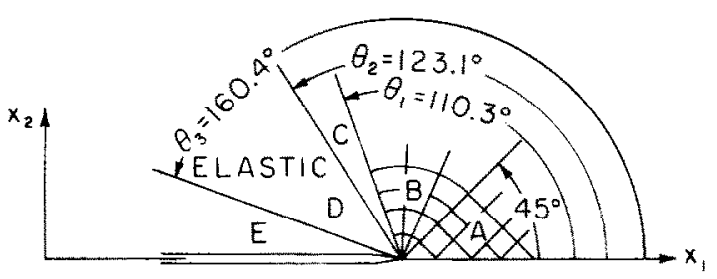

FIG. 5. The exact solution of the near-tip fields for a growing crack in material with $v=0.3$. This configuration results for general $v<0.5$ except that the angular locations of the sector boundaries $\left(\theta_{1}, \theta_{2}, \theta_{3}\right)$ vary with $v$, especially $O_{2}$, which approaches $\theta_{1}$ as $v \rightarrow 0.5$.

discussed later in this section, his boundary locations and deformation fields are incorrect due to an unjustified enforcement of full velocity continuity at $\theta=\pi / 4$.

Referring to Fig. 5, Regions $\mathrm{A}$ and $\mathrm{B}$ are $s_{33}-0$ plastic sectors of the constant stress and centered fan types, respectively. The general forms of their stress fields have been determined in Section 3.3.1. The stress field in Sector B is that corresponding to positive $\sigma_{r \theta}$ in (3.5), namely

$$
\begin{aligned}
& \sigma_{r \theta}=\mathrm{k}, \\
& \sigma_{r r}=\sigma_{\theta \theta}=\sigma_{33}=\mathrm{C}_{\mathrm{f}}-2 \mathrm{k} \theta,
\end{aligned}
$$

where $C_{f}$ is a constant, as yet undetermined. In Sector A all Cartesian stress components are constants, chosen to satisfy (4.3) - the Huber-Mises yield condition with $s_{33}=0$. Mode I symmetry requires $\sigma_{12}=0$ in this sector, so continuity of $\sigma_{r \theta}$ across the boundary between Sectors $A$ and $B$ requires this boundary to be at $\theta=\pi / 4$. Continuity of the remaining stress components across this boundary specifies the full stress field in Sector $A$ in terms of $C_{f}$, which remains undetermined:

$$
\begin{aligned}
& \sigma_{11}=\mathrm{C}_{\mathrm{f}}-\mathrm{k}(1+\pi / 2), \\
& \sigma_{22}=\mathrm{C}_{\mathrm{f}}+\mathrm{k}(1-\pi / 2), \\
& \sigma_{12}=0, \\
& \sigma_{33}=\left(\sigma_{11}+\sigma_{22}\right) / 2 .
\end{aligned}
$$

The velocity field in a moving centered fan which begins at $\theta=\pi / 4$ is given by (3.6), where continuity of $v_{\theta}$ across $\theta=\pi / 4$ was enforced, requiring the coefficient of the $\ln (\bar{R} / r)$ term of the expression for $v_{\theta}$ in the fan to vanish there since velocity components are less singular than $\ln r$ in Sector $A$ (see Section 5, where it is also proved that $v_{r}$ must possess a discontinuity across $\theta=\pi / 4)$. Note that the coefficients of the $\ln (\bar{R} / r)$ terms in these centered fan velocity expressions are thus completely determined. Therefore, full velocity continuity across $\theta_{1}$ permits $\mathrm{B}_{1}, \mathrm{~B}_{2}$ of Sector $\mathrm{C}$ to be completely specified in terms of $\theta_{1}$ :

$$
\begin{aligned}
& \mathrm{B}_{1}=-[(5-4 v) / \sqrt{ } 2](\mathrm{k} / E) \sin \theta_{1}, \\
& \mathrm{~B}_{2}=-[(5-4 v) / \sqrt{ } 2](\mathrm{k} / E)\left(\sqrt{ } 2-\cos \theta_{1}\right) .
\end{aligned}
$$

Furthermore, full velocity continuity across $\theta_{2}$ and $\theta_{3}$ requires that $\left(A_{1}, A_{2}\right)$ of Sector $D$ be equal to $\left(B_{1}, B_{2}\right)$ of Sector $C$ and to $\left(B_{1}, B_{2}\right)$ of Sector $E$, so that all three pairs of 
constants are identical and are given by (4.6). Thus the velocity components in Sectors $C, D$ and $E$ will all be of the form (3.2), with $\left(A_{1}, A_{2}\right)$ equal to $\left(B_{1}, B_{2}\right)$ of (4.6).

Sectors $\mathrm{C}$ and $\mathrm{E}$ are of the $s_{33} \neq 0$ non-singular plastic type. In such sectors, as analyzed in Section 3.2.2, the four stress components $\sigma_{r r}, \sigma_{r \theta}, \sigma_{\theta \theta}, \sigma_{33}$ are governed by four first-order ordinary differential equations: (2.4), (2.14) and (3.10). As noted, an analytical solution to this system for the general case of $\left(B_{1}, B_{2}\right)$ different from $(0,0)$ could not be found; hence a Runge-Kutta numerical scheme was employed to solve these equations in Sectors $\mathrm{C}$ and $\mathrm{E}$. To this end, the governing differential equations were recast in the following form

$$
\begin{aligned}
\sigma_{r \theta}^{\prime}= & 2\left(\sigma_{\theta \theta}-\sigma\right), \\
\sigma_{\theta \theta}^{\prime}= & -2 \sigma_{r \theta}, \\
\sigma^{\prime}= & 2 E s_{33}^{2}\left(\mathrm{~B}_{1} \cos \theta / \sin \theta+\mathrm{B}_{2}\right) /\left[4 s_{33}^{2}+4 v s_{33}\left(2 \sigma-2 \sigma_{\theta \theta}-s_{33}\right)\right. \\
& \left.+\left(2 \sigma-2 \sigma_{\theta \theta}-s_{33}\right)^{2}\right], \\
s_{33}^{\prime}= & -4\left(\sigma-\sigma_{\theta \theta}\right) \sigma^{\prime} /\left(3 s_{33}\right),
\end{aligned}
$$

where $\sigma \equiv\left(\sigma_{r r}+\sigma_{\theta \theta}\right) / 2$ and $\left(\mathbf{B}_{1}, \mathbf{B}_{2}\right)$ are given by (4.6). Noting that (4.7) are independent of hydrostatic stress, $\mathrm{C}_{\mathrm{f}}$ was set equal to zero for purposes of calculation and chosen later to satisfy the condition $\sigma_{\theta \theta}(\pi)=0$.

Sector D experiences purely elastic current response; as discussed in Section 3.1, the stress field in such a sector is given by (3.4), where in this case $\left(A_{1}, A_{2}\right)$ in those expressions equal $\left(B_{1}, B_{2}\right)$ given in (4.6).

Now, the determination of the three unknown boundary angles $\theta_{1}, \theta_{2}, \theta_{3}$ of Sectors $\mathrm{C}, \mathrm{D}$ and $\mathrm{E}$ in Fig. 5 for a specific $v$ value constitutes a two-parameter shooting problem, with the two independent parameters being $\theta_{1}$ and $\theta_{2}$. The following iteration procedure is employed : initial guesses are made for $\theta_{1}$ and $\theta_{2}$. Knowledge of $\theta_{1}$ permits full specification of stresses at that boundary from (4.4), with $C_{f}$ set equal to zero as discussed. Enforcing full stress continuity, a complete set of initial conditions is thus known to begin the numerical integration of (4.7) through Sector $C$; however, $s_{33}=0$ at $\theta_{1}$, meaning that (4.7) cannot be applied directly at $\theta_{1}$ but that Taylor series expansions of the stress components about $\theta_{1}$ are necessary to start the integration. These expansions for $\theta>\theta_{1}$ are, to $O\left[\left(\theta-\theta_{1}\right)^{3}\right]$ of (4.7),

$$
\begin{aligned}
\sigma_{r \theta} & =\mathrm{k}-\frac{(5-4 v)^{2} \mathrm{k}}{48(1-2 v)^{2} \sin ^{2} \theta_{1}}\left(\theta-\theta_{1}\right)^{4}+\ldots, \\
\sigma_{\theta \theta} & =-2 \mathrm{k} \theta_{1}-2 \mathrm{k}\left(\theta-\theta_{1}\right)+\frac{(5-4 v)^{2} \mathrm{k}}{120(1-2 v)^{2} \sin ^{2} \theta_{1}}\left(\theta-\theta_{1}\right)^{5}+\ldots, \\
\sigma & =-2 \mathrm{k} \theta_{1}-2 \mathrm{k}\left(\theta-\theta_{1}\right)+\frac{(5-4 v)^{2} \mathrm{k}}{24(1-2 v)^{2} \sin ^{2} \theta_{1}}\left(\theta-\theta_{1}\right)^{3}+\ldots, \\
s_{33} & =\frac{(5-4 v) \mathrm{k}}{3 \sqrt{2}(1-2 v) \sin \theta_{1}}\left(\theta-\theta_{1}\right)^{2}+\ldots
\end{aligned}
$$

So using (4.8), numerical integration of (4.7) is performed from $\theta=\theta_{1}+0.1^{\circ}$ to $\theta=\theta_{2}$ in 
increments of $0.01^{\circ}$. This results in values of all the stress components at $\theta_{2}$ : enforcing full stress continuity across that boundary enables the constants $C_{11}, C_{12}, C_{22}$ and $d$ of (3.4), the elastic stress field of Sector $D$, to be determined.

Thus (3.4) now fully specify the Sector $\mathrm{D}$ stress components in terms of angle $\theta$. These expressions are substituted into the yield condition (2.11), and $\theta$ is increased from $\theta_{2}$ until $(2.11)$ is satisfied. This determines the final elastic plastic boundary $\theta_{3}$. Full stress continuity across $\theta_{3}$ gives the initial values of the stresses necessary to numerically integrate (4.7) through Sector $F$ to $\theta=\pi$. (Note that Taylor expansions are not necessary at $\theta=\theta_{3}$, since $s_{33} \neq 0$ there.) The resulting values of $s_{33}$ and $\sigma_{r \theta}$ at $\theta=\pi$ are required by (3.10) and (4.2), respectively, to be zero; if they are not, new values for $\theta_{1}$ and $\theta_{2}$ are predicted by employing a Newton-Raphson iterative scheme, and the above procedure repeated until those conditions are satisfied. $C_{f}$ is then chosen to be the negative of the calculated $\sigma_{\theta \theta}(\pi)$ value, so that the second condition of (4.2) is satisfied. Once a solution has been found, the Runge-Kutta integration procedure through Sectors $C$ and $E$ is repeated with a different step size to assess the accuracy of the results; all results reported in this paper appear to be accurate to the number of significant figures presented.

An expression for asymptotic crack opening rate $\delta$ (where $\delta$ is the opening between upper and lower crack surfaces) is now easily obtained : since symmetry about the crack line requires $\delta=2 v_{2}(r, \pi)$, the expression (3.2) for $v_{2}$ in Sector E of Fig. 5 gives

$$
\delta=-2 \mathrm{~B}_{2} \dot{a} \ln (\tilde{R} / r)+2 \dot{a} \int\left[F_{22}(\theta) / \cos \theta\right] \mathrm{d} \theta,
$$

at small distances $r$ behind the crack tip. As discussed above, full velocity continuity across $\theta_{1}, \theta_{2}$ and $\theta_{3}$ means that $\left(B_{1}, B_{2}\right)$ of Sector $E$ are given by (4.6), so that (4.9) becomes

$$
\dot{\delta}=\sqrt{ } 2(5-4 v)\left(\sqrt{2}-\cos \theta_{1}\right)(\mathrm{k} / E) \dot{a} \ln (\bar{R} / r)+2 \dot{a} \int\left[F_{22}(\theta) / \cos \theta\right] \mathrm{d} \theta .
$$

4.3.2 Specific results for $v=0.3$. The above analysis was carried out for the specific case of $v=0.3$, and a solution having

$$
\theta_{1}=110.26^{\circ}, \quad \theta_{2}=123.13^{\circ}, \quad \theta_{3}=160.38^{\circ}, \quad \mathrm{C}_{\mathrm{f}}=5.6778 \mathrm{k}
$$

was found. [For the stationary crack Prandtl stress field, $C_{\mathrm{f}}=(1+3 \pi / 2) \mathrm{k}=5.71 \mathrm{k}$. ] This solution is illustrated in Fig. 5, which has been drawn to correspond to these angles. The sector types in Fig. 5 are described in Section 4.3.1, and the stress fields in Sectors A, B and D are given by the equations mentioned there, coupled with (4.11). As discussed, the stress fields in Sectors $C$ and $E$ were determined numerically; they are presented in Table 1.

The near-tip stress field of this solution is fully continuous for all angles $\theta$ about the crack tip; it is plotted in Fig. 6 along with the Prandt stress field of the stationary crack.

$\mathrm{GAO}(1980 \mathrm{~b}, 1981)$ has also analyzed the governing equations near a growing crack for $v<0.5$, assuming steady-state conditions (as opposed to the present treatment for general, nonsteady conditions). In the former work, he does not construct a complete asymptotic solution, but contends that the solution will have the same asymptotic configuration as the $v=0.5$ solution of Fig. 3 ; as discussed in Section 4.3.1, the solution cannot have this configuration for $v<0.5$ since full stress continuity is then impossible. 
TABLE 1. Stress field in Sector $C\left(110.26^{\circ}-123.13^{\circ}\right)$ and Sector $E$ $\left(160.38^{\circ}-180^{\circ}\right)$ of exact solution for $v=0.3$ (see Fig. 5 )

\begin{tabular}{ccccc}
\hline$\theta$ (degrees) & $\sigma_{1 \mathrm{l}} / \mathrm{k}$ & $\sigma_{22} / \mathrm{k}$ & $\sigma_{12} / \mathrm{k}$ & $\sigma_{33} / \mathrm{k}$ \\
\hline 110.26 & 2.479 & 1.179 & -0.7602 & 1.829 \\
114.35 & 2.438 & 0.9369 & -0.6608 & 1.703 \\
118.35 & 2.385 & 0.7231 & -0.5551 & 1.611 \\
123.13 & 2.308 & 0.5049 & -0.4256 & 1.538 \\
160.38 & 1.968 & -0.00199 & 0.00765 & 1.284 \\
164.37 & 1.978 & -0.00088 & 0.00415 & 1.237 \\
168.37 & 1.989 & -0.00026 & 0.00165 & 1.175 \\
172.37 & 1.996 & -0.00004 & 0.00038 & 1.105 \\
176.37 & 1.999 & 0.0 & 0.00002 & 1.037 \\
180.00 & 2.000 & 0.0 & 0.0 & 1.000 \\
\hline
\end{tabular}

Gao further contends that the plastic strain field in the constant stress sector ahead of a growing crack is proportional to $\ln r$; that this is not possible under conditions of full stress continuity is proved in Section 5 of the present paper. In the latter work, Gao rules out the configuration of Fig. 3 as a solution to the $v<0.5$ case, and he constructs a complete asymptotic solution having the same assemblage of sectors as in Fig. 5 ,

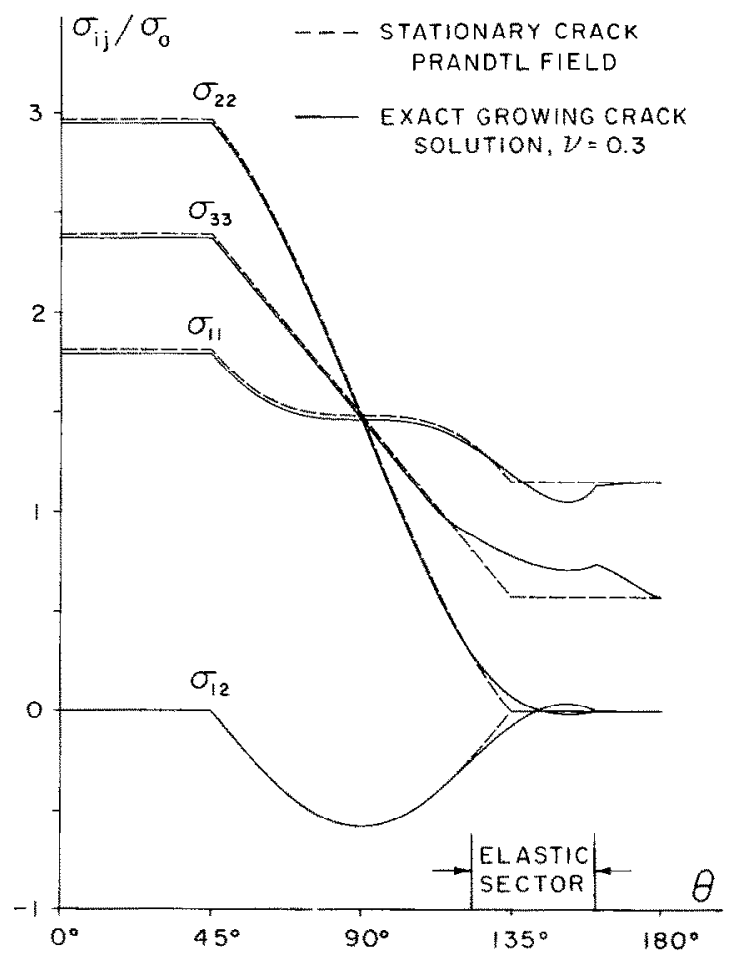

FiG. 6. Comparison of crack-tip stress states: dashed lines indicate the solution for a stationary crack (based on the Prandtl field of Fig. 2); solid lines display the exact growing crack solution (based on the field of Fig. 5) for $v=0.3$, which contains an elastic unloading sector. Stresses are normalized by tensile yield strength. 
although his boundary angles differ from ours for the same $v$ value. This difference arises at least in part due to an incorrect feature of his solution; namely, the erroneous conclusion arrived at in his prior paper that the plastic strain field ahead of the crack is proportional to $\ln r$. Further, the fracture criterion proposed by Gao is also based on this erroneous strain field ahead of the crack, while not accounting for the logarithmically singular plastic strain produced in the centered fan sector.

\section{Asymptotic Plastic Strain Field}

This section provides the derivations for the asymptotic strain field expressions for centered fan regions near stationary and growing cracks given by RICE (1982), who corrected similar results of RICE, DRUGaN and SHAM (1980). In addition, it is proved that the plastic strain field in the constant stress sector ahead of a growing plane strain crack (Sector A of Fig. 5) must be less singular (as $r \rightarrow 0$ ) than that of the adjoining centered fan sector (Sector B).

\subsection{Stationary crack}

The plastic strain field in the centered fan region near a stationary crack has the form

$$
c_{i j}^{\mathrm{p}}=L_{i j}(\theta) / r
$$

where only the $r \theta(=\theta r)$ component of $L_{i j}(\theta)$ is non-zero [and undetermined by the asymptotic analysis; RICE and TRACEY (1973) give some numerical results for $\left.L_{r \theta}(\theta)\right]$. That (5.1) results near a stationary crack is observed by setting $\dot{a}=0$ in (3.7), giving

$$
\begin{aligned}
& D_{r r}^{\mathrm{p}}=D_{\theta \theta}^{\mathrm{p}}=D_{3 i}^{\mathrm{p}}=0, \\
& D_{r \theta}^{\mathrm{p}}=(1 / 2 r)\left[\partial^{2} f(\theta, t) / \partial \theta^{2}+f(\theta, t)\right],
\end{aligned}
$$

to $O(1 / r)$ as $r \rightarrow 0$ since via $(2.10)$ (with $\Lambda=0) D_{i j}^{\mathrm{e}}=0$ to $O(1 / r)$ as $r \rightarrow 0$ when $\dot{a}=0$. The symbol $O(1 / r)$ is defined by

$$
|r O(1 / r)|<\infty \quad \text { as } \quad r \rightarrow 0 .
$$

Writing (5.2) in terms of Cartesian components,

$$
D_{i j}^{\mathrm{p}} \equiv \dot{\varepsilon}_{i j}^{\mathrm{p}}=(1 / 2 r) g_{i j}(\theta)\left[\partial^{2} f(\theta, t) / \partial \theta^{2}+f(\theta, t)\right]
$$

in a centered fan region near a stationary $\operatorname{crack} ; g_{i j}(\theta)$ are

$$
\begin{aligned}
& g_{11}(\theta)=-g_{22}(\theta)=-\sin 2 \theta, \\
& g_{12}(\theta)=g_{21}(\theta)=\cos 2 \theta, \\
& g_{3 i}(\theta)=0 .
\end{aligned}
$$

Integration of (5.3) with respect to time at a fixed material point gives, to $O(1 / r)$ as $r \rightarrow 0$,

$$
\varepsilon_{i j}^{\mathrm{p}}=(1 / 2 r) g_{i j}(\theta) \int_{-\infty}^{t_{0}}\left[\partial^{2} f(\theta, \tau) / \partial \theta^{2}+f(\theta, \tau)\right] \mathrm{d} \tau,
$$

which has the form (5.1), and we note that the only non-zero components of $g_{i j}(\theta)$ referred to the polar coordinate system are $g_{r \theta}(\theta)=g_{\theta r}(\theta)=e_{i} g_{i j} h_{j}=1$. 


\subsection{Growing crack}

The components of $r D_{i j}^{\mathrm{e}}$ in the centered fan near a growing crack (Sector B of Fig. 5) are given by the right side of (2.10), using (4.4):

$$
\begin{aligned}
& D_{r r}^{\mathrm{e}}=-2(2-v)(\mathrm{k} / E)(\dot{a} / r) \sin \theta, \\
& D_{\theta \theta}^{\mathrm{e}}=6 v(\mathrm{k} / E)(\dot{a} / r) \sin \theta, \\
& D_{33}^{\mathrm{e}}=-2(1-2 v)(\mathrm{k} / E)(\dot{a} / r) \sin \theta, \\
& D_{r \theta}^{\mathrm{e}}=D_{3 \alpha}^{\mathrm{e}}=0,
\end{aligned}
$$

to $O(1 / r)$ as $r \rightarrow 0$; from these, $D_{i j}^{p}\left(=D_{i j}-D_{i j}^{\mathrm{c}}\right)$ can be calculated using (3.7) and the plane strain condition $\left(D_{33}=D_{33}^{\mathrm{e}}+D_{33}^{\mathrm{p}}=0\right)$

$$
\begin{aligned}
D_{r r}^{\mathrm{p}} & =D_{\theta \theta}^{\mathrm{p}}=-(1-2 v)(\mathrm{k} / E)(\dot{a} / r) \sin \theta, \\
D_{r \theta}^{\mathrm{p}} & =D_{r \theta} \quad \text { of }(3.7), \\
D_{33}^{\mathrm{p}} & =2(1-2 v)(\mathrm{k} / E)(\dot{a} / r) \sin \theta, \\
D_{3 \alpha}^{\mathrm{p}} & =0 .
\end{aligned}
$$

Thus in terms of Cartesian components, the plastic strain rates in the centered fan are

$$
\begin{aligned}
D_{i j}^{\mathrm{p}}= & {[(5-4 v) /(2 \sqrt{ })](\mathrm{k} / E) g_{i j}(\theta)(\dot{a} / r) \ln (\bar{R} / r) } \\
& +(1-2 v)(\mathrm{k} / E)(\dot{a} / r)\left[3(1 / \sqrt{ } 2-\cos \theta) g_{i j}(\theta)+\left(3 \delta_{3 i} \delta_{3 j}-\delta_{i j}\right) \sin \theta\right] \\
& +(1 / 2 r) g_{i j}(\theta)\left[\partial^{2} f(\theta, t) / \partial \theta^{2}+f(\theta, t)\right],
\end{aligned}
$$

to $O(1 / r)$ as $r \rightarrow 0$, where $g_{i j}(\theta)$ are given in (5.4).

To prove that the rate of deformation $D_{i j}$ must be less singular than $1 / r$ as $r \rightarrow 0$ in Sector A of Fig. 5, assume as in Section 2.2 that $r \partial \sigma_{i j}(r, \theta, t) / \partial t \rightarrow 0$ as $r \rightarrow 0$. Then (2.8) can be written as

$$
\dot{\sigma}_{i j}=\left(\partial \sigma_{i j} / \partial \theta\right)\left(e_{2} \dot{a} / r\right)+O(1 / r) \quad \text { as } \quad r \rightarrow 0
$$

where $O(1 / r)$ is defined by

$$
r O(1 / r) \rightarrow 0 \quad \text { as } \quad r \rightarrow 0
$$

Taking the material time derivative of $(2.15)$ gives

$$
D_{33}^{\mathrm{p}}=-D_{33}^{\mathrm{e}}=-\left[\dot{\sigma}_{33}-v\left(\dot{\sigma}_{11}+\dot{\sigma}_{22}\right)\right] / E
$$

which, via (5.9), becomes

$$
D_{33}^{\mathrm{p}}=-\left[\sigma_{33}^{\prime}-v\left(\sigma_{11}^{\prime}+\sigma_{22}^{\prime}\right)\right](\sin \theta / E)(\dot{a} / r)+O(1 / r) \text { as } \quad r \rightarrow 0 .
$$

Employing (4.4) and (4.5), (5.12) gives for Sector B and Sector A, respectively, of Fig. 5

$$
\begin{aligned}
& \left.D_{33}^{\mathrm{p}}\right|_{\mathrm{B}}=2(1-2 v)(\mathrm{k} / E) \sin \theta(\dot{a} / r)+O(1 / r) \text { as } r \rightarrow 0, \\
& \left.D_{33}^{\mathrm{r}}\right|_{\mathrm{A}}=O(1 / r) \quad \text { as } \quad r \rightarrow 0 .
\end{aligned}
$$

The boundary between Sectors $A$ and $B$ is known from Section 4.3 .1 to be $\theta=\pi / 4$, rigorously only at $r=0$. Thus, this boundary (call it $S_{\mathrm{AB}}$ ) can be expressed for 
sufficiently small $r \geqslant 0$ as

$$
\theta=\pi / 4+m(r)
$$

where $m(r)$ is unknown except that $m(r) \rightarrow 0$ as $r \rightarrow 0$. Now, we require full stress continuity across $S_{\mathrm{AB}}$; using the plastic part of the Prandtl-Reuss flow rule

$$
D_{i j}^{\mathrm{p}}=\Lambda s_{i j}
$$

continuity of $s_{33}$ across $S_{\mathrm{AB}}$ demands

$$
s_{33}=\left.D_{33}^{\mathrm{p}}\right|_{\mathrm{B}} / \Lambda_{\mathrm{B}}=\left.D_{33}^{\mathrm{p}}\right|_{\mathrm{A}} / \Lambda_{\mathrm{A}} \text { on } S_{\mathrm{AB}}
$$

where the subscript A or B denotes the value of the subscripted quantity in Sector A or Sector B, respectively. Thus, rearranging and using (5.13) and (5.14),

$$
\begin{array}{r}
\Lambda_{\mathrm{A}} / \Lambda_{\mathrm{B}}=\left.D_{33}^{\mathrm{p}}\right|_{\mathrm{A}} /\left.D_{33}^{\mathrm{p}}\right|_{\mathrm{B}}=\frac{O(1 / r)}{2(1-2 v)(\mathrm{k} / E)(\dot{a} / r) \sin [\pi / 4+m(r)]+o(1 / r)} \\
\text { as } r \rightarrow 0 \text { along } S_{\mathrm{AB}}
\end{array}
$$

Since

$$
\sin [\pi / 4+m(r)]=\{\cos [m(r)]+\sin [m(r)]\} / \sqrt{2}
$$

and

$$
\begin{gathered}
\frac{\sin [m(r)]}{r} \sim \frac{m(r)}{r}=O(1 / r) \text { as } r \rightarrow 0, \\
\cos [m(r)] \rightarrow 1 \quad \text { as } \quad r \rightarrow 0,
\end{gathered}
$$

$(5.17)$ is

$$
\Lambda_{\mathrm{A}} / \Lambda_{\mathrm{B}}=\frac{O(1 / r)}{\sqrt{2(1-2 v)(\mathrm{k} / E)(\dot{a} / r)+o(1 / r)}} \text { as } r \rightarrow 0 \text { along } S_{\mathrm{AB}}
$$

Thus

$$
\Lambda_{\mathrm{A}} / \Lambda_{\mathrm{B}} \rightarrow 0 \quad \text { as } \quad r \rightarrow 0 \text { along } S_{\mathrm{AB}}
$$

Next enforce continuity of $s_{r r}$ across $S_{\mathrm{AB}} ;(5.15)$ requires

$$
s_{r r}=\left.D_{r r}^{\mathrm{p}}\right|_{\mathrm{B}} / \Lambda_{\mathrm{B}}=\left.D_{r r}^{\mathrm{p}}\right|_{\mathrm{A}} / \Lambda_{\mathrm{A}} \text { on } S_{\mathrm{AB}} .
$$

Rearranging and making use of (5.18),

$$
\left.D_{r r}^{\mathrm{p}}\right|_{\mathrm{A}} /\left.D_{r r}^{\mathrm{p}}\right|_{\mathrm{B}}=\Lambda_{\mathrm{A}} / \Lambda_{\mathrm{B}} \rightarrow 0 \quad \text { as } \quad r \rightarrow \text { along } S_{\mathrm{AB}}
$$

Now from (5.7) and (5.14),

$$
\left.D_{r r}^{\mathrm{p}}\right|_{\mathrm{B}}=-[(1-2 v) / \sqrt{ } 2](\mathrm{k} / E)(\dot{a} / r)+O(1 / r) \text { as } \quad r \rightarrow 0 \text { along } S_{\mathrm{AB}}^{+} ;
$$

thus (5.20) demands that

$$
\left.D_{r r}^{\mathrm{p}}\right|_{\mathrm{A}}=O(1 / r) \quad \text { as } \quad r \rightarrow 0 \text { along } S_{\mathrm{AB}}^{-} .
$$


Here, $S_{\mathrm{AB}}^{-}$denotes the limit of $\theta=\pi / 4+m(r)-\varepsilon$ as $\varepsilon \rightarrow 0 ; S_{\mathrm{AB}}^{+}$denotes that limit with $(+\varepsilon)$ replacing $(-\varepsilon)$. Further, from (2.7), (4.5) and (5.9),

$$
\left.D_{i j}^{\mathrm{e}}\right|_{\mathrm{A}}=O(1 / r) \text { as } r \rightarrow 0
$$

coupled with (5.22) shows

$$
\left.D_{r r}\right|_{\mathrm{A}}=\left.D_{r r}^{\mathrm{e}}\right|_{\mathrm{A}}+\left.D_{r r}^{\mathrm{p}}\right|_{\mathrm{A}}=O(1 / r) \text { as } r \rightarrow 0 \text { along } S_{\mathrm{AB}}^{-}
$$

Since $D_{r r}=\partial v_{r} / \partial r$, it is evident that $v_{r}$ in Sector A must be less singular than $\ln r$ as $r \rightarrow 0$ along $S_{\mathrm{AB}}^{-}$; comparing this result to the first equation of (3.6) yields the important conclusion that $v_{r}$ must undergo a jump across $S_{\mathrm{AB}}$ as $r \rightarrow 0$.

Now the plane strain condition $D_{33}=0$ applied to (2.7) specifies $\Lambda$ whenever $s_{33} \neq 0$; see (3.8). Applying this expression for $r>0$ in Sector A of Fig. 5, consideration of the $r$-dependence of the stress components (DRUGAN, 1982) shows that the most singular behaviour of $\Lambda$ possible in Sector $A$ is

$$
\Lambda_{\mathrm{A}} \sim 1 / r \text { as } r \rightarrow 0 .
$$

Coupling this result with (2.10) and (4.5) shows that the most singular behavior of $D_{i j}$ possible in Sector $\mathrm{A}$ is

$$
\left.D_{i j}\right|_{\mathrm{A}} \sim 1 / r \text { as } r \rightarrow 0 .
$$

But RICE (1982) showed that for the general field

$$
D_{i j}=\dot{a} F_{i j}(\theta) / r
$$

to satisfy plane strain compatibility, $F_{i j}(\theta)$ must meet the restriction

$$
F_{r r}(\theta)=\mathrm{C}_{1} \sin \theta+\mathrm{C}_{2} \cos \theta,
$$

where $C_{1}$ and $C_{2}$ are constants of integration. Applying this to Sector $A$, symmetry about the crack line requires $C_{1}=0$, and the result (5.24) demands $C_{2}=0$ also. Thus $F_{r r}(\theta) \equiv 0$. To determine the other components of $F_{i j}(\theta)$, note that (5.23) shows:

$$
\left.D_{i j}\right|_{\mathrm{A}}=\left.D_{i j}^{\mathrm{p}}\right|_{\mathrm{A}} \text {, }
$$

to $O(1 / r)$; thus (5.15) with (4.5) shows $F_{12}(\theta) \equiv 0$, and the plane strain condition requires $F_{33}(\theta) \equiv 0$ so (5.15) also shows $F_{11}(\theta)=-F_{22}(\theta)$. Finally, since

$$
F_{r r}(\theta)=F_{11}(\theta) \cos ^{2} \theta+2 F_{12}(\theta) \sin \theta \cos \theta+F_{22}(\theta) \sin ^{2} \theta,
$$

use of the preceding results shows $F_{11}(\theta) \equiv 0$. Therefore, we have proved that $F_{\xi j}(\theta) \equiv 0$, and hence that

$$
\left.D_{i j}^{\mathrm{p}}\right|_{\mathrm{A}}=O(1 / r) \text { as } r \rightarrow 0 .
$$

This important conclusion means that, by the arguments of Section 2.4.1, Sector A produces only bounded contributions to $\varepsilon_{i j}^{\mathrm{p}}$ as $r \rightarrow 0$, except possibly on $\theta=0$, where (5.29) demands that $\varepsilon_{i, j}^{\mathrm{p}}$ be less singular than $\ln r$ as $r \rightarrow 0$.

The jump in $v_{r}$ at the front boundary of the centered fan sector produces a step increase in plastic strain. To compute this increase, examine the kinematical conditions which must be satisfied at such a moving velocity discontinuity. Denote a jump in a 
field quantity, say $b(r, \theta, t)$, across $S_{\mathrm{AB}}$ as

$$
[[b]] \equiv \lim _{\varepsilon \rightarrow 0}\{b(r, \pi / 4+m(r)+\varepsilon, t)-b(r, \pi / 4+m(r)-\varepsilon, t)\} .
$$

For the material to maintain its integrity along $S_{\mathrm{AB}}, u_{\alpha}$ must satisfy

$$
\left[\left[u_{\alpha}\right]\right]=0 .
$$

Taking the time derivative of (5.31) following this moving boundary gives, as $r \rightarrow 0$,

$$
\left[\left[v_{\alpha}\right]\right]+(\dot{a} / \sqrt{2})\left(-h_{\beta}\right)\left[\left[\partial u_{\alpha} / \partial x_{\beta}\right]\right]=0 \text {. }
$$

From this, the radial velocity discontinuity as $r \rightarrow 0$ is

$$
\begin{aligned}
{\left[\left[v_{r}\right]\right] \equiv e_{\alpha}\left[\left[v_{\alpha}\right]\right] } & =(\dot{a} / \sqrt{ } 2) e_{\alpha} h_{\beta}\left[\left[\partial u_{\alpha} / \partial x_{\beta}\right]\right] \\
& =\sqrt{ } 2 \dot{a} e_{\alpha} h_{\beta}\left[\left[\frac{1}{2}\left(\partial u_{\alpha} / \partial x_{\beta}+\partial u_{\beta} / \partial x_{\alpha}\right)\right]\right],
\end{aligned}
$$

since

$$
\left[\left[\partial u_{\beta} / \partial x_{\alpha}\right]\right] e_{\alpha}=\left[\left[\partial u_{\beta} / \partial r\right]\right]=0
$$

via $(5.31)$, so $(5.33)$ reduces to

$$
\left[\left[v_{r}\right]\right]=\sqrt{2 a}\left[\left[\varepsilon_{r \theta}\right]\right] \text { as } r \rightarrow 0 .
$$

Full stress continuity across $S_{\mathrm{AB}}$ means that $\left[\left[\varepsilon_{i j}^{\mathrm{e}}\right]\right]=0$, so the result of a jump in $v_{r}$ is to produce a jump in $\varepsilon_{r \theta}^{p}$ :

$$
\left[\left[\varepsilon_{r \theta}^{\mathrm{p}}\right]\right]=\left[\left[v_{r}\right]\right] /(\sqrt{ } 2 \dot{a}) \quad \text { as } \quad r \rightarrow 0 .
$$

Since $v_{r}$ must be less singular than $\ln r$ as $r \rightarrow 0$ along $S_{\mathrm{AB}}$ in Sector $\mathrm{A}$, the most singular term of $\left[\left[v_{r}\right]\right]$ is given by the term proportional to $\ln r$ in the first expression of (3.6); thus $(5.35)$ is

$$
\left[\left[\varepsilon_{r \theta}^{\mathrm{p}}\right]\right]=(1 / 2)(5-4 v)(\mathrm{k} / E) \ln (\bar{R} / r)+o(\ln r) \text { as } r \rightarrow 0 .
$$

The plastic strain at a material point in the centered fan can be obtained, when $a$ is increasing continuously with applied loading, by integrating (5.8) and adding the strain accumulated due to the velocity discontinuity, (5.36). Integration of (5.8) is effected (RICE, 1974) by using $\theta=e_{2} \dot{a} / r$ to substitute for $\dot{a}$ and by replacing $r$ with $y / \sin \theta$ since the $y$-coordinate remains fixed as the crack grows past a material point; the resulting expression is integrated with respect to $\theta$, beginning at $\theta=\pi / 4$. The final expression is

$$
\varepsilon_{i j}^{\mathrm{p}}=[(5-4 v) /(2 \sqrt{2})](\mathrm{k} / E) G_{i j}(\theta) \ln (\bar{R} / r)+O(\ln r),
$$

where

$$
\begin{aligned}
G_{11}(\theta) & =-G_{22}(\theta)=-2 \sin \theta, \\
G_{12}(\theta) & =G_{21}(\theta)=\ln [\tan (\theta / 2) / \tan (\pi / 8)]+2[\cos \theta-(1 / \sqrt{2})], \\
G_{3 i} & =0 .
\end{aligned}
$$

Note that (5.37) gives the plastic strain at a material point which has passed through the constant stress region (Sector A of Fig. 5) and has current location $(r, \theta)$ in the centered fan (Sector B); the plastic strain accumulated by the material point during its passage 
through Sector A was proved to be at most $O(\ln r)$, and therefore does not contribute to the first term of (5.37).

Alternatively, for an equivalent plastic shear strain, $\gamma^{\mathbf{p}}$, defined as (HILL, 1950)

$$
\dot{\gamma}^{\mathbf{p}}=\left(2 D_{i j}^{\mathbf{p}} D_{i j}^{p}\right)^{1 / 2},
$$

using (5.7) one integrates (5.39) in the fan in the manner described above and adds the $\gamma^{\mathrm{p}}$ accumulated discontinuously at the front boundary of the fan to obtain

$$
\gamma^{\mathrm{p}}=(5-4 v)(\mathrm{k} / E)\{1+(1 / \sqrt{ } 2) \ln [\tan (\theta / 2) / \tan (\pi / 8)]\} \ln (\bar{R} / r)+O(\ln r)
$$

at a material point in the fan.

\section{A Ductile Crack Growth Criterion}

The asymptotic deformation field results can be used as the basis for a ductile crack growth criterion, as proposed by RICE and SORENSEN (1978) and extended by RICE, DRUGAN and SHAM (1980). The crack opening rate expression (4.10) derived in the exact $v<0.5$ analysis has the same form as that derived by RICE, DRUGAN and SHAM (1980),

$$
\dot{\delta}=\beta\left(\sigma_{0} / E\right) \dot{a} \ln (\bar{R} / r)+\dot{A}
$$

where $\sigma_{0}=\sqrt{ } 3 \mathrm{k}$ is the tensile yield strength, $\dot{A}$ is a parameter undetermined by the asymptotic analysis but homogeneous of degree one in $\dot{a}$ and in the rate of applied load increase, and their exact $v=0.5$ analysis gave $\beta=4.385$. (RICE, Drugan and SHam (1980) also performed an approximate asymptotic analysis for $v=0.3$, from which they obtained $\beta=5.08$. This approximate analysis contained an error, however, as discussed in Section 3.2.1 and in the Appendix which gives the details of a correct approximate asymptotic analysis.) Comparison of (4.10) and (6.1) gives $\beta$ for the present exact $v<0.5$ analysis,

$$
\beta=(\sqrt{ } 6 / 3)(5-4 v)\left(\sqrt{ } 2-\cos \theta_{1}\right),
$$

so using the results of the specific solution of Section 4.3.2,

$$
\beta=5.462 \text { for } v=0.3 \text {. }
$$

This corresponds very closely to the $\beta$ value determined from the numerical finite element solution of plane strain crack growth under small scale yielding conditions of Sham (1979) as reported in Rice, Drugan and Sham (1980), where $\beta=5.4$ was given as a representative value of data which varied within the range $\pm 1.4 \%$ of $\beta=5.4$. A recent, more refined numerical finite-element simulation of growth by SHAM (1982), discussed subsequently, is also supportive and gives $\beta=5.46$ as an average of numerical data varying within a $\pm 0.3 \%$ range.

Now since (4.10) has the same form as (6.1), the full development of a crack growth criterion from (6.1) by RICE, DRUGAN and SHAM (1980) can be applied to the present $v=0.3$ exact solution. In particular, they show that (6.1) may be rewritten as

$$
\dot{\delta}=\alpha \dot{J} / \sigma_{0}+\beta\left(\sigma_{0} / E\right) \dot{a} \ln (R / r),
$$

where $\bar{R}$ has been replaced by a new length parameter $R$ which, along with the 
dimensionless quantity $\alpha$, is undetermined by the asymptotic analysis ; $J$ is the far-field value of the $J$-integral, taken in surrounding elastic material. For continuous crack growth $(\mathrm{d} J / \mathrm{d} a$ finite), integration of (6.4) results in

$$
\delta=\beta\left(\sigma_{0} / E\right) r \ln (\rho / r)
$$

very near the crack tip, where

$$
\rho \equiv R \exp \left[1+(\alpha / \beta)\left(E / \sigma_{0}^{2}\right)(\mathrm{d} J / \mathrm{d} a)\right] .
$$

Expression (6.5) shows that there is no opening at a growing crack tip, but that $\mathrm{d} \delta / \mathrm{d} r$ is infinite there. It is further evident from (6.5) that the parameter $\rho$ fully characterizes the crack profile near the tip ; thus, RICE, DRUGAN and SHAM (1980) proposed $\rho=$ constant as a crack growth criterion, which is the requirement that a geometrically similar neartip profile be maintained for continued growth. They also investigated a crack growth criterion based on the attainment of a critical accumulated plastic strain, $\gamma_{c}^{p}$, by all material points within a certain small characteristic distance above and below the crack tip. It was shown that the resulting differential equation governing growth is identical in form to the one resulting from the $\rho=$ constant criterion, and hence would be expected to provide qualitatively similar conclusions.

The parameters $\alpha$ and $R$ were estimated by SHAm $(1979,1982)$, where two independently calculated finite-element solutions for a growing crack under smallscale yielding conditions were employed. In the earlier solution, crack growth was simulated by repetition of a two-step process : first, the crack tip nodal force was incrementally unloaded while maintaining constant external loading; then the external loading was increased with the new crack length held fixed. The finite elements employed wcre constant strain triangles, arranged in groups of quadrilaterals [sec Nagtegaal, Parks and RICE (1974)] in order to relieve the artificial mesh locking effects which arise under nearly-incompressible conditions.

The more recent calculation (SHAM, 1982) simulates crack growth in a more realistic fashion; namely, the external loading is increased simultaneously with the release of the crack tip nodal force. This calculation employs four-noded quadrilateral elements, and artificial mesh locking effects are handled via the specialization of a modified variational principle of Nagtegaal, PARKs and Rice (1974) to the element type employed. The far-field boundary conditions appropriate to small-scale yielding are enforced by embedding the finite-element mesh in the analytically-simulated surrounding continuum. This procedure increases the ratio of the maximum plastic zone size obtained to the size of the smallest element employed; specifically, the ratio is 100 for this calculation, as compared to 50 for the earlier one (SHAM, 1979).

The results of these two solutions indicate that for the modest range of tearing modulus $T\left[\equiv\left(E / \sigma_{0}^{2}\right) \mathrm{d} J / \mathrm{d} a\right]$ examined, $0 \leqslant T \lesssim 20, \alpha$ is approximately independent of crack growth history [to within $2 \%$ variation for the $\alpha$ values estimated in the SHAM (1982) solution] and has the value

$$
\alpha \simeq 0.6 \text {. }
$$

As opposed to the SHAM (1979) solution which indicated that the value of $\alpha$ was roughly the same for a growing crack as for a stationary crack, the SHAM (1982) solution indicates that $\alpha$ is approximately $5 \%$ less for the growing crack than its estimated stationary crack value. 
The parameter $R$ was estimated by SнAм (1979) to be given by

$$
R=s E J / \sigma_{0}^{2}
$$

with $s \simeq 0.23$ and approximately constant over the limited amounts of crack growth simulated. The new calculation of SHAM (1982) suggests that $s$ is not constant, but varies within the approximate range $0.11-0.13$. However, the source of this variation is not well established, and the precise implications for $R$ remain unclear.

Dean and Hutchinson (1980) and Parks, Lam and MCMeEking (1981) have also performed finite element calculations for a growing crack under small scale yielding conditions. In both of these investigations a special finite-element procedure was employed to calculate directly the steady-state solution, and estimates of $\beta$ and $R$ were made. DEAN and HutCHINSON (1980) reported that $\beta=4.28$ and $s=0.78$ would best fit the four computed values of the crack opening nearest the tip in their results, while $s=0.31$ would best fit the same data if $\beta$ were fixed at 5.08. PARKs, LAM and MCMEEKING (1981) reported the values $\beta=5.808, s=0.1824$ as representing the best fit to their results. Thus, further work appears necessary to clarify the precise value of $R$ and its possible dependence on growth history.

For crack growth under small scale yielding conditions, the criterion $\rho=$ constant means that (6.6), with (6.7) and (6.8), results in a differential equation which specifies the behaviour of $J$ in terms of $a$ necessary for continued growth

$$
\mathrm{d} . J / \mathrm{d} a=(\beta / \alpha)\left(\sigma_{0}^{2} / E\right) \ln \left[\rho \sigma_{0}^{2} /(\operatorname{se} E . J)\right] .
$$

Here, e is the natural logarithm base, and (6.9) is to be integrated subject to the initial condition $J=J_{1 \mathrm{c}}$ at growth onset. The quantity $\rho$ is theorized to be a material parameter, so from (6.9) materials which can exhibit stable crack growth under small scale yielding conditions must have

$$
\rho \geqslant \operatorname{se} E J_{\mathrm{Ic}} / \sigma_{0}^{2}
$$

since if the value of $\mathrm{d} J / \mathrm{d} a$ calculated from (6.9) at the $J_{\text {Ic }}$ point is negative, immediately unstable crack growth is expected. For materials which satisfy (6.10), inspection of (6.9) reveals that $\mathrm{d} J / \mathrm{d} a$ has an initially positive value, so that $J$ increases with continued crack growth, causing $\mathrm{d} J / \mathrm{d} a$ to decrease continually to zero. At $\mathrm{d} J / \mathrm{d} a=0$, crack growth persists with no further increase in $J$; from (6.9), this 'steady-state' $J$ is

$$
J_{\mathrm{ss}}=(1 / \mathrm{se}) \rho \sigma_{0}^{2} / E \text {. }
$$

Thus, stable crack growth under small scale yielding conditions in a material with $v=0.3$ is governed by (6.9) and (6.11), with $\alpha$ and $\beta$ specified by (6.7) and (6.3), respectively. Rice, DRUGan and Sham (1980) show how this fracture criterion can be phrased in terms of alternate parameters to $J_{\text {Ic }}$ and $\rho$, permitting various interpretations more identifiable with other (e.g., microstructural) viewpoints.

HERMANN and RICE (1980) investigated this model by comparing the predicted $J$ vs $a$ curves with those obtained from experiments on a high-strength AISI-4140 steel $\left(\sigma_{\text {yield }}\right.$ $=1173 \mathrm{MPa}, \sigma_{\text {utt. }}=1327 \mathrm{MPa}$ ), selected for its ability to provide several millimeters of stable crack growth under plane strain conditions in a typical specimen before the occurrence of general yield. The test geometry was that of a deeply-cracked bend specimen, so the 'deformation theory' value of the $J$-integral for this configuration was 
determined from the measurements; crack growth ranging from approximately 5 to $10 \mathrm{~mm}$ was produced prior to attainment of general yield. The theoretical $J$ vs $a$ curves (calculated using $\beta=5.08, \alpha=0.65$ and $s=0.23$ ) were found to agree reasonably with the experimentally obtained curves for the full range of sub-general yield stable crack growth, the parameter values giving the best fit to the data being $J_{\mathrm{Ic}}=35 \mathrm{kN} \mathrm{m}^{-1}$ and $\rho=7.2 \mathrm{~mm}$. The latter implies by (6.11) that $J_{\mathrm{ss}}=80 \mathrm{kN} \mathrm{m}^{-1}$ which gives a level of an apparent plateau in the experimental curves directly prior to general yielding.

\section{ACKNOWLEDGEMENTS}

This study was primarily supported by the U.S. Department of Energy under Contract DEAC02-80ER/10556 with Brown University, with minor additional support (JRR) from the NSF Materials Rescarch Laboratory at Harvard University. Helpful discussions with Dr. Y. C. GAO of the Harbin Shipbuilding Engineering Institute, Harbin, China, are gratefully acknowledged.

\section{REFERENCES}

Cherepanov, G. P.

1974

DEAN, R. H. and HutChinson, J. W.

Drugan, W. J.

GAO, Y.-C.

HermanN, L. and Rice, J. R.

HiLl, R.

HuTCHINSON, J. W.

LeVy, N., MarCal, P. V., OSTERGREN, W. J. and

RICE, J. R.

MCCLintock, F. A.
Mechanics of Brittle Fracture (in Russian) Nauka, Moscow. (English translation by A. L. Peabody, edited by R. DEWIT and W. C. COOLEY). McGraw-Hill, 1979.

1980 Fracture Mechanics: Twelfth Conference, ASTMSTP 700, p. 383. American Society for Testing and Materials, Philadelphia.

1982 Radial Dependence of Stress and Deformation Fields Near the Tip of a Growing Plane Strain Tensile Crack in an Elastic-Ideally Plastic Solid. Ph.D. Thesis, Ch. II, Brown University.

1980a Acta mech. sin. 1, 48 (in Chinese).

1980b Elastic-Plastic Fields at Crack Tips in PerfectlyPlastic Medium (reported at 15th ICTAM, Toronto, 17-23 August 1980); Acta mech. sin., in press.

1981 The Influence of Compressibility on the Elastic Plastic Field of a Growing Crack (reported at ASTM Second International Symposium on Elastic-Plastic Fracture Mechanics, Philadelphia, 6-10 October 1981, submitted for publication).

1980 Met. Sci. 14, 285.

1950 The Mathematical Theory of Plasticity, pp. 334 340. Clarendon Press, Oxford.

1968 J. Mech. Phys. Solids 16, 337.

1971 Int. J. Fracture Mech. 7, 143.
Fracture: An Advanced Treatise (edited by $\mathrm{H}$. LiEBowITZ), Vol. 3, Engineering Fundamentals and Environmental Effects, p. 47. Academic Press, New York. 
Nagtegaal, J. C., Parks, D. M. and RICE, J. R.

Parks, D. M., Lam, P. S. and McMeEking, R. M.

RICE, J. R.

Rice, J. R., Drugan, W. J. and SHAM, T.-L.

Rice, J. R. and Rosengren, G. F. RICE, J. R. and SORENSEN, E. P. Rice, J. R. and Tracey, D. M.

SHAM, T-L.

1982

SI.FPYAN, L. I
1974 Comp. Meth. Appl. Mech. Engr. 4, 153.

1982 Advances in Fracture Research (Proceedings of the Fifth International Conference on Fracture, Cannes, April 1981), (edited by D. FrancoIs et al.), Vol. 5, p. 2607. Pergamon Press, Oxford.

1967 Fatigue Crack Propagation, ASTM-STP 415, p. 247. American Society for Testing and Materials, Philadelphia.

1968 Fracture: An Advanced Treatise (edited by H. Liebowitz), Vol. 2, Mathematical Fundamentals, p. 191. Academic Press, New York.

1974 Mechanics and Mechanisms of Crack Growth (Proceedings of Conference organized by the Physical Metallurgy Centre of British Steel Corporation held at Churchill College, Cambridge, 4 6 April 1973) (edited by M. J. MAY), p. 14. British Steel Corporation, London.

1982 Mechanics of Solids (edited by H. G. Hopkins and M. J. Sewell), p. 539. Pergamon Press, Oxford.

1980 Fracture Mechanics: Twelfth Conference, ASTMSTP 700, p. 189. American Society for Testing and Materials, Philadelphia.

1968 J. Mech. Phys. Solids 16, 1.

1978 Ibid. 26, 163.

1973 Numerical and Computer Methods in Structural Mechanics (Proceedings of ONR Symposium), (edited by S. J. Fenves et al.) p. 585. Academic Press, New York.

A Finite-Element Analysis of Quasi-Static Crack Growth in an Elastic Perfectly Plastic Solid. Sc.M. Thesis, Brown University.

A Finite Element Study of the Asymptotic Near-Tip Fields for Mode I Plane Strain Cracks Growing Stably in Elastic Ideally Plastic Solids. Proc. ASTM Second Int. Symp. on Elastic-Plastic Fracture Mechanics, Philadelphia, 1981, to be published.

1974 Izv. Akad. Nauk. SSSR. Mekhanika Tverdogo Tela 9, 57. (Translated from Russian.)

APPENDIX

An APPRoXimate ASYMPTOTIC ANALYSIS FOR $v<0.5$

RiCE, DRUGAN and Sham (1980) quoted the results of an approximate asymptotic analysis of the ficlds ncar a growing crack for $v<0.5$, stating that the details of that analysis would be presented in a future publication. This Appendix provides those details, correcting an erroneous limit calculation that their analysis contained as discussed in Section 3.2.1.

The approximate asymptotic analysis to be discussed differs from the exact $v<0.5$ analysis of 
Section 4.3 due to the approximation made that

$$
s_{33} \rightarrow 0 \text { as } r \rightarrow 0
$$

at all angles of plastic response near the advancing crack tip. That this is a good approximation will be shown explicitly here; Rice, DRUGAN and SHAM (1980) motivated its assumption by noting that a plastic strain singularity is expected at a plane strain crack tip and hence that (A.1) is rigorously valid at all angles of plastic response at which singular plastic strain is produced. Further motivation for assuming (A.1) is the fact that $s_{33} \equiv 0$ everywhere when $v=0.5$.

Making the approximation that (A.1) holds at all angles of plastic response about the crack tip, and hence that the Huber-Mises yield condition reduces to the in-plane form (4.3), permits only two possible types of asymptotic plastic sector: the centered fan and constant stress types described in Section 3.2.1. Thus the near-tip field must be comprised of at most three sector types, the third possible type being the currently elastic sector (Section 3.1); this is the same situation as for the exact solution of the field near a growing crack when $v=0.5$. The configuration representing the solution to that case, Fig. 3 , is therefore postulated to result for the present $v<0.5$ approximate analysis, with the elastic sector boundary angles $\theta_{1}, \theta_{2}$ being as yet undetermined. The analysis of the stresses in Sectors A and B of Fig. 3 is identical to that described in Section 4.3.1, so that the angle of the boundary between these sectors is $\theta=\pi / 4$ (independent of $v$ ), and their stress fields are (4.5) for Sector $A$ and (4.4) for Sector B, with $C_{f}$ initially unknown. Sector $D$ is assumed to be of the constant stress type ; enforcing the crack flank boundary conditions (4.2) completely determines the stress field in this sector

$$
\begin{aligned}
& \sigma_{11}=2 \mathrm{k}, \\
& \sigma_{33}=\mathrm{k}, \\
& \sigma_{12}=\sigma_{22}=0 .
\end{aligned}
$$

Finally, appropriate continuity conditions must be specified across $\theta_{1}$ and $\theta_{2}$, namely full stress continuity and full velocity continuity. The remaining unknowns in the problem are the elastic sector constants $\mathrm{A}_{1}, \mathrm{~A}_{2}, \mathrm{C}_{11}, \mathrm{C}_{12}, \mathrm{C}_{22}$ and $\mathrm{d}[\mathrm{see}(3.2)-(3.4)]$, and $\theta_{1}, \theta_{2}$ and $\mathrm{C}_{\mathrm{f}}$. Thus the problem is overspecified, since there are 10 conditions restricting 9 unknowns (velocity continuity across $\theta=\theta_{2}$ does not constrain these unknowns). This overspecification is the result of assuming (A.1) to be true at all angles of plastic response about the crack tip; the explicit effects of this assumption are that continuity of $\sigma_{33}$ across $\theta=\theta_{2}$ cannot be enforced, and that the elastic sector stress field is not everywhere below yield. (When $v=0.5$, these conditions are satisfied automatically, and the present formulation is exact.) These facts indicate that the true solution for $v<0.5$ must contain angular sectors in which $s_{33} \neq 0$ at $r=0$, as shown in Section 4.3. Nevertheless, assumption (A.1) allows for a much simpler analysis to determine approximate values for the locations of the rear fan boundary and the elastic-plastic reloading boundary, $C_{f}$, the coefficient $\beta$ of (6.1) and the near-tip stress field, for a given value of $v<0.5$.

Enforcing full stress (except $\sigma_{33}$ at $\theta_{2}$ ) and velocity continuity results in the elastic sector constants being given by

$$
\begin{aligned}
\mathrm{C}_{11} & =2 M \mathrm{k}-\mathrm{A}_{1}\left[4 \ln \left|\sin \theta_{2}\right|+\cos 2 \theta_{2}\right]-\mathrm{A}_{2}\left(2 \theta_{2}+\sin 2 \theta_{2}\right), \\
\mathrm{C}_{12} & =-\mathrm{A}_{1}\left(2 \theta_{2}+\sin 2 \theta_{2}\right)+\mathrm{A}_{2} \cos 2 \theta_{2}, \\
\mathrm{C}_{22} & =\mathrm{A}_{1} \cos 2 \theta_{2}-\mathrm{A}_{2}\left(2 \theta_{2}-\sin 2 \theta_{2}\right), \\
\mathrm{d} & =(1-2 v)\left(\mathrm{C}_{\mathrm{f}}-2 \mathrm{k} \theta_{1}\right),
\end{aligned}
$$

where $M=4\left(1-v^{2}\right) / E$ and $\left(\mathrm{A}_{1}, \mathrm{~A}_{2}\right)=\left(\mathrm{B}_{1}, \mathrm{~B}_{2}\right)$ of (4.6). Furthermore, in contrast to the exact analysis of Section 4.3 .1 , there results a closed form expression for $C_{f}$,

$$
\begin{aligned}
\mathrm{C}_{\mathrm{r}} / \mathrm{k}=1+2 \theta_{1}-(\sqrt{ } 2 / 4)\left[(5-4 v) /\left(1-v^{2}\right)\right]\left[\sin \theta_{1} \ln \left|\sin \theta_{1} / \sin \theta_{2}\right|\right. \\
\left.+\left(\sqrt{ } 2-\cos \theta_{1}\right)\left(\theta_{1}-\theta_{2}\right)\right] .
\end{aligned}
$$


and a pair of coupled transcendental equations for $\theta_{1}$ and $\theta_{2}$,

$$
\begin{aligned}
& -\sin \theta_{1}\left[2 \ln \left|\sin \theta_{1} / \sin \theta_{2}\right|+\cos 2 \theta_{1}-\cos 2 \theta_{2}\right] \\
& \quad+\left(\cos \theta_{1}-\sqrt{2}\right)\left(\sin 2 \theta_{1}-\sin 2 \theta_{2}\right)+4 \sqrt{ } 2\left[\left(1-v^{2}\right) /(5-4 v)\right]\left(1+\sin 2 \theta_{1}\right)=0, \\
& \sin \theta_{1}\left[2\left(\theta_{1}-\theta_{2}\right)+\sin 2 \theta_{1}-\sin 2 \theta_{2}\right] \\
& \quad+\left(\cos \theta_{1}-\sqrt{ } 2\right)\left(\cos 2 \theta_{1}-\cos 2 \theta_{2}\right)+4 \sqrt{ } 2\left[\left(1-v^{2}\right) /(5-4 v)\right] \cos 2 \theta_{1}=0 .
\end{aligned}
$$

It is easily shown that an expression for $\delta$ of the form (6.1) results from the present configuration, and enforcing velocity continuity across $\theta=\theta_{2}$ requires that $\beta$ be given in terms of $\theta_{1}$ and $v$ by (6.2), the same expression resulting from the exact $v<0.5$ analysis.

Now, for a given value of $v,(A .5)$ are solved by a Newton-Raphson scheme; $\mathrm{C}_{\mathrm{f}}$ is then directly available from (A.4), and $\beta$ from (6.2).

Equations (A.5) have some interesting features. For values of $v<0.5$, these equations have three sets of solutions $\left(\theta_{1}, \theta_{2}\right)$. One set is always $\theta_{1}=\theta_{2}=135^{\circ}$, corresponding to the Prandtl field of Fig. 1. This solution must be ruled out for a growing crack since, as discussed in Section 4.2.2, the resulting deformation field contains a line of negative plastic work production. Another solution set to (A.5) for $v<0.5$ is dependent on $v$ but corresponds to a very narrow elastic sector (e.g., for $v=0.3$, this solution is $\theta_{1} \simeq 127^{\circ}, \theta_{2}=135^{\circ}$ ). This solution must also be ruled out on the basis of negative plastic work production, which occurs in the plastic reloading sector following the elastic sector. Fortunately, a solution set to (A.5) for which only positive plastic work is produced (corresponding to a much wider elastic sector) can be found for each $v$; this is the solution desired. For $v=0.5$, (A.5) have only two solution sets: $\theta_{1}=\theta_{2}=135^{\circ}$, and the exact result quoted in Section 4.2 .2 of $\theta_{1}=112.08^{\circ}, \theta_{2}=162.10^{\circ}$.

Table 2 shows the accuracy of this approximate analysis by comparing the values of $\theta_{1}, \theta_{2}, C_{\mathrm{f}}$ and $\beta$ obtained, to $\theta_{1}, \theta_{3}, C_{\mathrm{f}}$ and $\beta$, respectively, from the exact analysis of Section 4.3.2, for $v=0.3$.

TABLE 2. Comparison of exact vs approximate growing crack solutions for $v=0.3$

\begin{tabular}{lllcc}
\hline & $\beta$ & \multicolumn{1}{c}{$\mathrm{C}_{\mathrm{f}} / \mathrm{k}$} & $\begin{array}{c}\text { Rear boundary } \\
\text { of fan }\end{array}$ & $\begin{array}{c}\text { Plastic reloading } \\
\text { sector boundary }\end{array}$ \\
\hline $\begin{array}{l}\text { Exact analysis } \\
\begin{array}{l}\text { Approximate analysis } \\
\text { of Appendix A }\end{array}\end{array}$ & 5.462 & 5.6778 & $110.26^{\circ}$ & $160.38^{\circ}$ \\
\hline
\end{tabular}

\section{Authors' Note}

The requirement of full stress continuity between asymptotic angular sectors for the growing crack, stated as an assumption in Section 4.3.1, has recently been proved by W. J. DruGAN and $\mathbf{J}$. R. RICE (Drucker Anniversary Volume: Mechanics of Material Behavior, edited by G. J. DVORAK and R. T. SHIELD, Elsevier, Amsterdam, in press) as a special case of a general analysis of the continuity restrictions on quasistatically moving surfaces in elastic-plastic solids. In addition, their analysis derives specific conditions on the stress state at such a moving surface if discontinuities in tangential velocity and shear strain are to be allowed. We note that the discontinuities in velocity and strain which occur in the growing crack solution of the present paper do so across a surface (the boundary hetween the constant stress and centered fan regions, Sectors A and B of Fig. 5, as $r \rightarrow 0$ ) whose stress state meets these specific conditions. 


\title{
ERRATUM
}

\section{ASYMPTOTIC ANALYSIS OF GROWING PLANE STRAIN TENSILE CRACKS IN ELASTIC-IDEALLY PLASTIC SOLIDS}

\author{
W. J. Drugan, J. R. Rice and T. L. Sham
}

J. Mech. Phys. Solids 30, 447 (1982)

The Publisher regrets that a section of the text for the above paper was printed incorrectly. All expressions printed as $O(1 / r)$ and $O(\ln r)$, from equation (5.9) on p. 463 to equation (5.40) on p. 467 inclusive, should be printed as $o(1 / r)$ and $o(\ln r)$ respectively. 